\title{
A well-balanced shock-capturing hybrid finite volume-finite difference numerical scheme for extended 1D Boussinesq models
}

\author{
M. Kazolea ${ }^{\mathrm{a}}$, A.I. Delis ${ }^{\mathrm{b}}$ \\ ${ }^{a}$ Environmental Engineering Department, Technical University of Crete, University Campus, Chania, Crete 73100, Greece \\ ${ }^{b}$ Department of Sciences, Division of Mathematics, Technical University of Crete, University Campus, Chania, Crete 73100, Greece
}

\begin{abstract}
A formally fourth-order well-balanced hybrid finite volume/difference (FV/FD) numerical scheme for approximating the conservative form of two $1 \mathrm{D}$ extended Boussinesq systems is presented. The FV scheme is of the Godunov type and utilizes Roe's approximate Riemann solver for the advective fluxes along with well-balanced topography source term upwinding, while FD discretizations are applied to the dispersive terms in the systems. Special attention is given to the accurate numerical treatment of moving wet/dry fronts. To access the performance and applicability, by exposing the merits and differences of the two formulations, the numerical models have been applied to idealized and challenging experimental test cases. Special attention is paid in comparing both Boussinesq models to the nonlinear shallow water equations (NSWE) in the simulation of the experimental results. The outcomes from this work confirm that, although the NSWE can be sufficient in some cases to predict the general characteristics of propagating waves, the two Boussinesq- models provided considerable more accurate results for highly dispersive waves over increasing water depths.
\end{abstract}

Keywords: Boussinesq-type equations, finite volume/difference method, solitary waves, wave breaking

\section{Introduction}

In the last two decades mathematical and numerical modeling of free surface flows in realistic environments has been one of the most interesting and active research fields in coastal engineering. Important issues one has to consider include, the validity of a mathematical model in near-shore zones as well as in deeper waters, wave breaking, transitions between sub and supercritical flows, frequency dispersion and accurate numerical treatment of natural topographies and wetting/drying processes. To this end, depth averaged models have gained a lot of popularity, in terms of applicability and development, with the nonlinear shallow water equations (NSWE) being one of the most applied models falling in this category. Written in conservative form, for mass and momentum, the one-dimensional (1D) NSWE read as

$$
\begin{aligned}
& H_{t}+(H u)_{x}=0, \\
& (H u)_{t}+\left(H u^{2}+\frac{1}{2} g H^{2}\right)_{x}=-g H b_{x}-\tau
\end{aligned}
$$

Email addresses: mskazolea@isc.tuc.gr (M. Kazolea), adelis@science.tuc.gr (A.I. Delis) 
where the subscripts $x \in \mathbb{R}$ and $t>0$ denote partial derivatives with respect to space and time, $H(x, t)=$ $\eta(x, t)+d(x)$ is the total fluid depth (with $\eta(x, t)$ being the free surface elevation and $d(x)$ the depth below the still water level), $u(x, t)$ is the horizontal depth-averaged velocity, $g$ is the gravitational constant, $b(x)$ is the bed topography function and $\tau$ is a term that models friction viscous dissipation effects. For a well-posed problem we add to system (1) and (2) some initial conditions

$$
H(x, 0)=H_{0} \quad \text { and } \quad u(x, 0)=u_{0}
$$

where $H_{0}$ and $u_{0}$ are given functions on $\mathbb{R}$, as well as boundary conditions. Equations (1) and (2) represent a system of (hyperbolic) conservation laws with source terms (also called balanced laws) that can describe the propagation of very long waves under the hydrostatic pressure assumption.

The NSWE have been employed widely to model wave propagation and runup, see for example [5, 6, $15,16,23,30,34,35,43,44]$ among many others. In addition, numerical models that utilize the NSWE have the ability to represent breaking waves as propagating bores or hydraulic jumps (i.e. shock discontinuities) $[16,30]$. When dealing with flow discontinuities volume conservation becomes an important issue. Numerically, conservative Godunov-type finite volume (FV) methods based on Riemann solvers have the advantage of solving the integral form of the nonlinear equations as fully conservative schemes with intrinsic shock capturing properties as well as with correctly incorporating the bed topography and treating accurately advancing wet/dry fronts $[4,15,17,23,29,34,47,48]$. With regard to the topography discretization, considerable progress has been made in devising numerical schemes for computing the topography source term within the FV framework. Several numerical and mathematical treatments have been proposed in the literature for balancing the flux gradient and the source terms, in order to properly compute stationary or almost stationary solutions. This property is known as well-balancing and is currently a very active subject of research, we refer to [15] and references therein. The second important problem is the appearance of dry areas, due to initial conditions or as a result of the water motion. As such, the necessity to handle wetting and drying moving boundaries (e.g. shoreline motion [3]) is a challenge that has attracted much attention. Several approaches have been proposed in different models and numerical schemes. In [43] using the NSWE equations and in [31] for Boussinesq-type equations an extrapolation technique was used to allow the models to handle such moving boundaries. Other techniques have been developed as to avoid dealing with wet/dry interfaces by excluding the dry cells from the computational domain [4, 6], by artificially wetting dry cells [23] and by modeling the shore as porous or slotted [25].

Although the models utilizing the NSWE appear to be able to model important aspects of the wave breaking process and the general characteristics of the runup process they are not appropriate for deeper waters where frequency dispersion effects become more important than nonlinearity. On the other hand, Boussinesq-type equations introduce dispersion terms and are more suitable in water where dispersion begins to have an effect on the free surface. Under the assumption that nonlinearity and frequency dispersion are weak, and in the same order of magnitude, Peregrine [37] derived the so called standard Boussinesq equations for variable depth using the free surface displacement and the depth averaged velocity as dependent variables. The standard Boussinesq equations, written in terms of the depth averaged velocity, break down when the depth, $d$, is greater than one fifth of the equivalent deep water wavelength, $L$, and as such are limited to relative shallow water. In addition the weakly nonlinear assumption limits the largest wave height that can be accurately modeled. Considerable effort has been made in the past two decades to extend the validity and applicability of the standard Boussinesq equations to deeper water depths (or to shorter waves). As such, several alternative formulations with improved dispersive properties were presented. The extended Boussinesq formulations of Madsen and Sørensen (MS) [33] and Nwogu [36] have received, probably, the most attention in recent years for modeling wave propagation from deep to shallow water. The two approaches have identical dispersion characteristics closely relating to linear wave theory leading to a 
dispersion relation that can be interpreted as a Padé approximant of the exact dispersion relation. These extended models give a more accurate representation of the phase and group velocities in intermediate water (with water depth to wavelength ratio $d / L$ up to $1 / 2$ ). However, further improvements have been derived by including high-order nonlinear and dispersion terms (see [32], [27] for a review) which in turn are more difficult to integrate and thus may require substantial computational effort.

Many numerical schemes have been developed to compute approximate solutions for Boussinesq-type equations. Until recently most of them are based on the finite difference (FD) method but the use of the finite element (FE) method has increased in the past few years, see [18] for a review. However, the FV method requires significantly less computational effort than the FE method while non-linear advection terms can be more easily treated when compared to the FD method. Very recently, hybrid solutions which combine the FV and FD method have been introduced for Boussinesq-type equations as to incorporate the flexibility and shock-capturing capabilities of the FV method into dispersive wave models. This approach is particularly useful for short and long wave interactions as the solution can be easily turned into entirely FV solution of the NSWE by removing the higher order Boussinesq terms if needed. In [21] a fourth-order hybrid FV/FD model for the MS model was developed by employing a MUSCL-type scheme with Roe's Riemann solver on the FV part of the equations. In addition, the fourth-order predictor-corrector time stepping from [49] was employed and the topography slopes were discretized using the surface gradient method (SGM) [52]. However, no specific wet/dry front and wave breaking treatments were considered. In [2] and [45, 46] similar schemes to that presented in [21] were developed to solve the MS equations by employing the HLL Riemann solver instead and wave breaking criteria were introduced resulting in a hybrid Boussinesq/NSWE model. Both the MS and Nwogu's models, but in non-conservative form, were numerically solved and compared in [40] utilizing again the HLL solver and the SGM in the predictor corrector MUSCL-Hancock scheme. Again, no specific wet/dry front and wave breaking treatments were considered. In [39] a fifthorder in space and a fourth-order in time (from [49]) hybrid scheme was presented for Nwogu's model along with a new eddy viscosity breaking mechanism, based on the variations of the momentum flux. Other notable hybrid approaches, but for different Boussinesq-type models, can be found for example in [12$14,19,20,26,31,41]$.

In the light and spirit of these recent developments, in the present work both Nwogu's model and the Madsen and Sørensen (MS) one are formulated in the conservative form of the NSWE. Both models are numerically solved employing a fourth-order Godunov-type FV technique, based on the approximate Riemann solver of Roe with the topography source term discretized in an upwind manner as to provide a well-balanced scheme. An accurate conservative treatment of moving wet/dry fronts and shoreline motion is integrated in the proposed scheme. As such, no special algorithms need to be implemented, e.g. extrapolation or exclusion of dry cells, to accurately compute shoreline movements. In addition, a specific wave breaking treatment is included and tested here, following [45, 46], also for Nwogu's model. This study has twofold; the development and application of a hybrid FV/FD well-balanced scheme to Nwogu's and MS equations, expressed in conservation form, as well as, the comparison of the results obtain from the two models in order to determine their differences (if any) to practical numerical applications for idealized and, more important, experimental benchmark data. Finally, the two Boussinesq models are compared also to the non-dispersive NSWE for the experimental test cases.

\section{Dispersive Model Equations}

The two systems of extended Boussinesq equations presented next are applicable for irregular wave propagation on a slowly varying bathymetry from deeper to shallow water. Both models are rewritten here 
into a conservation law form and as such they can be numerically approximated by a hybrid FV/FD scheme. Each model is different in the form and arrangement of dispersive terms.

\subsection{Nwogu's Boussinesq formulation}

Nwogu [36] derived a system of equations using the velocity $u \equiv u_{a}$ at an arbitrary distance from a still water level $z_{a}$ as the velocity variable, instead of the commonly used depth-averaged velocity $u$. An optimum value of $z_{a}=-0.531 d$ was used so that the dispersion properties of the system most closely approximate those defined by linear wave theory, making the equations applicable to a wider range of water depths compared to the classical Boussinesq equations. The extended equations of Nwogu consist of a continuity and a momentum equation in the form:

$$
\begin{array}{r}
\eta_{t}+(H u)_{x}+\left[\left(\frac{z_{a}^{2}}{2}-\frac{d^{2}}{6}\right) d u_{x x}+\left(z_{a}+\frac{d}{2}\right) d(d u)_{x x}\right]_{x}=0, \\
u_{t}+u u_{x}+g \eta_{x}+z_{a}\left[\frac{z_{a}}{2} u_{x x}+(d u)_{x x}\right]_{t}+\tau=0,
\end{array}
$$

We re-derive the equations following [39]. Since the topography $b$ is unchanged in time, it holds that:

$$
\eta_{t}=H_{t}, \quad \eta_{x}=H_{x}-d_{x}, \quad \text { and } \quad g H \eta_{x}=\left(\frac{g H^{2}}{2}\right)_{x}+g H b_{x} .
$$

Including relationships from (6) in equations (4) and (5) we can re-write them in vector conservative form:

$$
\mathbf{U}_{t}+\mathbf{F}(\mathbf{U})_{x}=\mathbf{S}(\mathbf{U})
$$

where now $\mathbf{U}$ is the vector of then new variables, $\mathbf{F}$ is the flux vector and $\mathbf{S}$ is the source term, with

$$
\mathbf{U}=\left[\begin{array}{c}
H \\
P^{\star}
\end{array}\right], \mathbf{F}(\mathbf{U})=\left[\begin{array}{c}
H u \\
H u^{2}+\frac{1}{2} g H^{2}
\end{array}\right], \mathbf{S}(\mathbf{U})=\mathbf{S}_{\mathbf{b}}+\mathbf{S}_{\mathbf{f}}+\mathbf{S}_{\mathbf{d}}=\left[\begin{array}{c}
0 \\
-g H b_{x}
\end{array}\right]+\left[\begin{array}{c}
0 \\
-\tau
\end{array}\right]+\left[\begin{array}{c}
-\psi_{C} \\
-u \psi_{C}+\psi_{M}
\end{array}\right]
$$

in which

$$
\begin{aligned}
& P^{\star}=H u+H z_{a}\left(\frac{z_{a}}{2} u_{x x}+(d u)_{x x}\right), \\
& \psi_{M}=H_{t} z_{a}\left(\frac{z_{a}}{2} u_{x x}+(d u)_{x x}\right), \\
& \psi_{C}=\left[\left(\frac{z_{a}^{2}}{2}-\frac{d^{2}}{6}\right) d u_{x x}+\left(z_{a}+\frac{d}{2}\right) d(d u)_{x x}\right]_{x} .
\end{aligned}
$$

$P^{\star}$ is the velocity-like function and contains all time-derivatives in the momentum equation and a part of the dispersion terms, while $H_{t}$ can be explicitly defined by the continuity equation, so the dispersion terms $\psi_{C}$ and $\psi_{M}$ contain only spatial derivatives.

\subsection{Madsen and Sørensen's (MS) Boussinesq formulation}

The equations derived by Madsen and Sørensen [33] are: 


$$
\begin{aligned}
\eta_{t} & +(H u)_{x}=0 \\
(H u)_{t} & +\left(H u^{2}\right)_{x}+g H \eta_{x}+\tau \\
& +\left[-\left(B+\frac{1}{3}\right) d^{2}(H u)_{x x t}-B g d^{3} \eta_{x x x}-d d_{x}\left(\frac{1}{3}(H u)_{x t}+2 B g d \eta_{x x}\right)\right]=0
\end{aligned}
$$

where $B$ is a free parameter that determines the dispersion properties of the system. A value of $B=1 / 15$ is used here [33]. We re-write the above equations following [21, 45], in order to obtain a conservative formulation. As in Nwogu's equations, it is assumed that the topography is not changing with time so including relations from (6) in (11) and (12) leads to the conservative form (7), where now

$$
\mathbf{U}=\left[\begin{array}{c}
H \\
\tilde{P}^{\star}
\end{array}\right], \quad \mathbf{F}(\mathbf{U})=\left[\begin{array}{c}
H u \\
H u^{2}+\frac{1}{2} g H^{2}
\end{array}\right], \quad \mathbf{S}(\mathbf{U})=\mathbf{S}_{\mathbf{b}}+\mathbf{S}_{\mathbf{f}}+\tilde{\mathbf{S}}_{\mathbf{d}}=\left[\begin{array}{c}
0 \\
-g H b_{x}
\end{array}\right]+\left[\begin{array}{c}
0 \\
-\tau
\end{array}\right]+\left[\begin{array}{c}
0 \\
\psi
\end{array}\right]
$$

in which

$$
\begin{aligned}
\tilde{P}^{\star} & =H u-\left(B+\frac{1}{3}\right) d^{2}(H u)_{x x}-\frac{1}{3} d d_{x}(H u)_{x} \quad \text { and } \\
\psi & =-B g d^{3} \eta_{x x x}-2 d^{2} d_{x} B g \eta_{x x} .
\end{aligned}
$$

The source term $\mathbf{S}(\mathbf{U})$ includes again the bottom slope $\mathbf{S}_{\mathbf{b}}$, friction $\mathbf{S}_{\mathbf{f}}$ and part of the dispersive terms is included in $\tilde{\mathbf{S}}_{\mathbf{d}}$. The term $\tilde{P}^{\star}$ contains all time derivatives in the momentum equation and the dispersion term $\psi$ contains only spatial derivatives.

For the friction term $\tau$, in both models, the Manning empirical formula, $\tau=g H S_{f}$, is used here, where

$$
S_{f}=n_{m}^{2} \frac{u|u|}{H^{4 / 3}}
$$

with $n_{m}$ being Manning's friction coefficient.

Both Nwogu's and MS Boussinesq models describe weakly non-linear and weakly dispersive water waves in variable water depth and were derived under the assumption that

$$
\epsilon:=A / d<<1, \quad \mu^{2}:=d^{2} / L^{2}<<1, \quad S:=\epsilon / \mu^{2}=O(1)
$$

where $\epsilon$ and $\mu$ are standard nondimensional parameters for a scale analysis of long waves, $S$ is the Stokes (or Ursell) number, with $A$ being a typical wave amplitude and $L$ a characteristic wavelength. Both sets of equations provide accurate linear dispersion and shoaling characteristics for values of $k d$ up to 3 (intermediate water depths), where $k$ is the wave number and $k d$ is essentially a scale of the value of $\mu$, providing a correction of $O\left(\mu^{2}\right)$ to the shallow water theory. However, the property of the Padé approximant is such that the resulting dispersion relations for both models may be interpreted as being accurate through $O\left(\mu^{4}\right)$ or display an extended validity range. By retaining $O\left(\mu^{2}\right)$ terms in the derivation of the models some vertical variations in the horizontal velocity are included even though the explicit appearance of the vertical coordinate has been removed from the continuity and momentum equation by integration. In the absence of the dispersion terms, by omitting all $\mu$ terms, both models satisfy exactly the NSWE (1) and (2). Written in the above conservative form, both Nwogu's and MS set of equations have an identical advective flux vector $\mathbf{F}(\mathbf{U})$ to that of the NSWE. The use of the conservative form of the equations as well as the application of a conservative FV numerical scheme ensures proper handling of flows characterized by discontinuities. 
An important property of (7), related to the topography source term, is that the system admits non-trivial steady-states. A particular elementary solution that provides a benchmark for many numerical schemes is the so-called flow (or lake) at rest solution that is easily obtained assuming $u=0$ and $H+b=D(x, 0)=D_{0}$ constant in the wet region of the computational domain $\Omega \subset \mathbb{R}$. Then, we get the exact solution

$$
\begin{aligned}
u & =0 \quad \forall x \in \Omega \text { and } t \geq 0, \\
H(x, t) & =\left\{\begin{array}{ll}
D_{0}-b(x) & \text { if } b(x)<D_{0}, \\
0 & \text { otherwise, }
\end{array} \quad t \geq 0 .\right.
\end{aligned}
$$

This solution represents flow at rest taking into account the existence of wet/dry transitions as well, and is of importance for the derivation of FV discretizations presented in this study. In numerical applications we require the above steady state solutions to be preserved exactly. Schemes that satisfy this property are usually referred to as well-balanced. If a numerical scheme is not well-balanced, even very small perturbations of steady-state solutions may lead to numerical-induced errors, which would make the resolution of long waves impossible due to their small amplitude during their propagation to deeper waters. More precisely, a numerical scheme that preserves exactly (16) and (17) is said to verify the $\mathcal{C}$-property [1] and if wet/dry transitions exist the extended $C$-property [10].

\section{The hybrid numerical scheme}

The hybrid FV/FD numerical scheme used to solve the equations of Nwogu and MS is formally fourthorder in space and time. We use the FV formulation for the discretization of the advetive part, $\mathbf{F}(\mathbf{U})$, of the equations and the bed source term, $\mathbf{S}_{\mathbf{b}}$, and the FD formulation for the corresponding dispersive terms.

\subsection{FV spatial discretization (for leading order terms)}

The FV formulation imposes conservation laws in a control volume which reduces here to a one dimensional cell. Let us consider a control volume $\left[x_{i-1 / 2}, x_{i+1 / 2}\right] \times\left[t^{n}, t^{n+1}\right]$ of dimensions $\Delta x=x_{i+1 / 2}-x_{i-1 / 2}$ and $\Delta t=t^{n+1}-t^{n}$. A Cartesian mesh is used in which the index $i$ represent a particular cell centroid where the average quantities of the conserved variables are nominally stored. The approximate cell average of $\mathbf{U}$ in $C_{i}=\left[x_{i-1 / 2}, x_{i+1 / 2}\right]$ at time $t^{n}$ is denoted as $\mathbf{U}_{i}^{n}$ i.e. $\mathbf{U}_{i}^{n}=\frac{1}{\Delta x} \int_{C_{j}} \mathbf{U}\left(x, t^{n}\right) d x$.

High-order accuracy in the calculation of numerical fluxes is achieved by constructing (left and right) cell interface values using a fourth-order MUSCL-type extrapolation scheme, given by Yamamoto et al. [50], prior to the application of the numerical flux, see also [21, 40, 45]. This fourth-order reconstruction is performed to the variables of total water depth $H$, velocity $u$, and also, in this work, to the topography $b$ as follows, for the $(i+1 / 2)$ cell interface:

$H_{i+1 / 2}^{L}=H_{i}+\frac{1}{6}\left[\varphi\left(r_{1}\right) \Delta^{*} H_{i-1 / 2}+2 \varphi\left(\frac{1}{r_{1}}\right) \Delta^{*} H_{i+1 / 2}\right] \quad$ and $\quad H_{i+1 / 2}^{R}=H_{i+1}-\frac{1}{6}\left[2 \varphi\left(r_{2}\right) \Delta^{*} H_{i+1 / 2}+\varphi\left(\frac{1}{r_{2}}\right) \Delta^{*} H_{i+3 / 2}\right]$

where $\varphi$ is the van Leer nonlinear slope limiter function, [29],

$$
\begin{gathered}
\varphi\left(r_{i}\right)=\frac{r_{i}+\left|r_{i}\right|}{1+\left|r_{i}\right|} \text { with } r_{1}=\frac{\Delta^{*} H_{i+1 / 2}}{\Delta^{*} H_{i-1 / 2}}, \quad r_{2}=\frac{\Delta^{*} H_{i+3 / 2}}{\Delta^{*} H_{i+1 / 2}} \text { and } \\
\Delta^{*} H_{i+1 / 2}=\Delta H_{i+1 / 2}-\frac{1}{6}\left(\Delta \bar{H}_{i+3 / 2}-2 \Delta \bar{H}_{i+1 / 2}+\Delta \bar{H}_{i-1 / 2}\right)
\end{gathered}
$$


where

$$
\begin{aligned}
\Delta \bar{H}_{i-1 / 2} & =\operatorname{minmod}\left(\Delta H_{i-1 / 2}, \Delta H_{i+1 / 2}, \Delta H_{i+3 / 2}\right), \\
\Delta \bar{H}_{i+1 / 2} & =\operatorname{minmod}\left(\Delta H_{i+1 / 2}, \Delta H_{i+3 / 2}, \Delta H_{i-1 / 2}\right), \\
\Delta \bar{H}_{i+3 / 2} & =\operatorname{minmod}\left(\Delta H_{i+3 / 2}, \Delta H_{i-1 / 2}, \Delta H_{i+1 / 2}\right), \\
\Delta H_{i+1 / 2} & =H_{i+1}-H_{i},
\end{aligned}
$$

and the minmod limiter given as: $\operatorname{minmod}(a, b, c)=\operatorname{sign}(a) \max (|a|, 2 \operatorname{sign}(a) b, 2 \operatorname{sign}(a) c)$.

After integration of (7) over each computational cell $C_{i}$ the semidiscrete form for the advective and topography terms can be represented as

$$
\frac{\Delta \mathbf{U}_{i}^{n}}{\Delta t}=-\frac{1}{\Delta x}\left[\mathbf{F}_{i+1 / 2}^{n}-\mathbf{F}_{i-1 / 2}^{n}\right]+\frac{1}{\Delta x} \Delta \mathbf{S}_{\mathbf{b} i}^{n}=-\frac{1}{\Delta x} \Delta \mathbf{F}_{i}^{n}+\frac{1}{\Delta x} \Delta \mathbf{S}_{\mathbf{b} i}^{n},
$$

where $\mathbf{F}_{i \pm 1 / 2}^{n}$ and $\Delta \mathbf{S}_{\mathbf{b} i}^{n}$ are the numerical fluxes (at each cell interface) and the numerical topography source respectively. The numerical fluxes in (23) can be evaluated solving the Riemann problem at the cell interfaces. In this study the approximate Riemann solver of Roe [38] is utilized along with an upwind discretization for the topography source and are briefly reproduced here for the sake of completeness and in order to present some proposed modifications. We remark here that, modified versions of Roe's solver, e.g. [11, 19], as well as other approximate Riemann solvers, e.g. of the HLL-type [47], can be applied for the construction of the numerical flux. The choice of Roe's solver in this work is justified by its wide popularity and applicability and more importantly by the well established numerical treatment of the topography source terms in order to satisfy the $C$-property, which can be easily incorporated in this solver. By denoting with $\Delta_{i+1 / 2}(\cdot)=(\cdot)_{i+1 / 2}^{R}-(\cdot)_{i+1 / 2}^{L}$ and $\mathbf{F}_{i+1 / 2}^{R, L}=\mathbf{F}\left(\mathbf{U}_{i+1 / 2}^{R, L}\right)$, the numerical fluxes in (23) are defined as

$$
\mathbf{F}_{i+1 / 2}=\frac{1}{2}\left(\mathbf{F}_{i+1 / 2}^{R}+\mathbf{F}_{i+1 / 2}^{L}\right)-\frac{1}{2}\left|\mathbf{A}_{i+\frac{1}{2}}\right| \Delta_{i+1 / 2} \mathbf{U}=\frac{1}{2}\left(\mathbf{F}_{i+1 / 2}^{R}+\mathbf{F}_{i+1 / 2}^{L}\right)-\frac{1}{2}\left[\mathbf{X}|\mathbf{\Lambda}| \mathbf{X}^{-1}\right]_{i+1 / 2} \Delta_{i+1 / 2} \mathbf{U}
$$

where $\mathbf{A}_{i+1 / 2}$ is the Roe average Jacobian matrix, $\frac{\partial \mathbf{F}(\mathbf{U})}{\partial \mathbf{U}}$, which can be diagonalized, by the right and left eigenvector matrices $\mathbf{X}_{i+1 / 2}$ and $\mathbf{X}_{i+1 / 2}^{-1}$, with $\boldsymbol{\Lambda}_{i+1 / 2}$ being the diagonal matrix with the eigenvalues in the diagonal. With the Roe average matrix between $\mathbf{U}_{i+1 / 2}^{R}$ and $\mathbf{U}_{i+1 / 2}^{L}$ given as

$$
\mathbf{A}_{i+\frac{1}{2}}=\left[\begin{array}{cc}
0 & 1 \\
\tilde{c}_{i+1 / 2}^{2}-\tilde{u}_{i+1 / 2}^{2} & 2 \tilde{u}_{i+1 / 2}
\end{array}\right]
$$

where the average velocity $\tilde{u}$ and celerity $\tilde{c}$ are:

$$
\tilde{u}_{i+1 / 2}=\frac{u^{L} \sqrt{H^{L}}+u^{R} \sqrt{H^{R}}}{\sqrt{H^{L}}+\sqrt{H^{R}}} \quad \text { and } \quad \tilde{c}_{i+1 / 2}=\sqrt{g \frac{H^{R}+H^{L}}{2}}
$$

the eigenvalues of $\mathbf{A}_{i+1 / 2}$ are $\lambda_{i+1 / 2}^{1,2}=\tilde{u}_{i+1 / 2} \pm \tilde{c}_{i+1 / 2}$, with corresponding eigenvectors $\mathbf{r}_{i+1 / 2}^{1}=\left[1, \lambda_{i+1 / 2}^{1}\right]^{\mathrm{T}}$ and $\mathbf{r}_{i+1 / 2}^{2}=\left[1, \lambda_{i+1 / 2}^{2}\right]^{\mathrm{T}}$.

As it has been demonstrated in [1,24], an upwind discretization scheme should be also used for the bed topography source term, $\mathbf{S}_{\mathbf{b}}$, to avoid non-physical oscillations in the solution by satisfying the $C$-property. To satisfy this, the discrete topography source term should balance the corresponding non-zero flux terms, so it must be linearized in the same way and evaluated in the same state (Roe-averaged state) as the flux. The 
upwind discretization of the source term in (23) provides the following two terms (in-going contributions) that are added to the corresponding computational cell giving,

$$
\Delta \mathbf{S}_{\mathbf{b} i}=\mathbf{S}_{\mathbf{b} i+1 / 2}^{-}+\mathbf{S}_{\mathbf{b} i-1 / 2}^{+}
$$

where

$$
\mathbf{S}_{\mathbf{b} i+1 / 2}^{+}=\frac{1}{2}\left[\mathbf{X}\left(\mathbf{I}+\boldsymbol{\Lambda}^{-1}|\mathbf{\Lambda}|\right) \mathbf{X}^{-1}\right]_{i+1 / 2} \widetilde{\mathbf{S}}_{\mathbf{b} i+1 / 2} \quad \text { and } \quad \mathbf{S}_{\mathbf{b} i+1 / 2}^{-}=\frac{1}{2}\left[\mathbf{X}\left(\mathbf{I}-\boldsymbol{\Lambda}^{-1}|\mathbf{\Lambda}|\right) \mathbf{X}^{-1}\right]_{i+1 / 2} \widetilde{\mathbf{S}}_{\mathbf{b i + 1 / 2}}
$$

with

$$
\widetilde{\mathbf{S}}_{\mathbf{b} i+1 / 2}=\left[\begin{array}{c}
0 \\
-g \frac{H^{L}+H^{R}}{2}\left(b^{R}-b^{L}\right)
\end{array}\right]_{i+1 / 2} .
$$

From the above, the advective numerical flux terms should now equal the source term for a flow at rest in (23), i.e., $\Delta \mathbf{F}_{i}^{n}=\Delta \mathbf{S}_{\mathbf{b} \text { i }}^{n}$, which should give at each mesh cell: $u=0$ and $b^{R}-b^{L}=-\left(H^{R}-H^{L}\right)$. While this equilibrium holds for the first order scheme, i.e. $\mathbf{U}^{L}=\mathbf{U}_{i}$ and $\mathbf{U}^{R}=\mathbf{U}_{i+1}$, this is not the case for the fourth-order MUSCL-type discretization. As such, and following [24, 35] a correction term, $\mathbf{S}_{\mathbf{b}}^{\star}$, should be included to the topography source discretization (righthand side of (27)) for maintaining the correct balance. This term reads as,

$$
\mathbf{S}_{\mathbf{b}}^{\star}\left(\mathbf{U}_{i+1 / 2}^{L}, \mathbf{U}_{i-1 / 2}^{R}\right)=\left[\begin{array}{c}
0 \\
-g \frac{H_{i-1 / 2}^{R}+H_{i+1 / 2}^{L}}{2}\left(b_{i-1 / 2}^{R}-b_{i+1 / 2}^{L}\right)
\end{array}\right]
$$

and can be considered as the topography source integral approximated over the mesh cell which vanishes for a first order scheme as $b_{i-1 / 2}^{R}=b_{i+1 / 2}^{L}=b_{i}$, while for flow at rest we have in addition: $b_{i+1 / 2}^{L}-b_{i-1 / 2}^{R}=$ $-\left(H_{i+1 / 2}^{L}-H_{i-1 / 2}^{R}\right)$. Hence, in the present work, we have for the full topography source discretization applied

$$
\Delta \mathbf{S}_{\mathbf{b} i}=\mathbf{S}_{\mathbf{b} i+1 / 2}^{-}+\mathbf{S}_{\mathbf{b} i-1 / 2}^{+}+\mathbf{S}_{\mathbf{b}}^{\star}\left(\mathbf{U}_{i+1 / 2}^{L}, \mathbf{U}_{i-1 / 2}^{R}\right) .
$$

This corrected discretization for the topography enforces the well-balanced property in (23) for the steady solution in (17) when $b(x)<D_{0} \forall x \in \Omega$, i.e. totally wetted computational domain.

\subsubsection{Wet-dry front treatment and mass conservation}

In wetting and drying zones, e.g. in the near-shore regions, water inundates or recedes and the difficulty in numerical modeling dry areas relates to the obvious fact that the models are only defined for wet regions. As such, and following [17], several issues have to be addressed to accurately model wet/dry transitions.

Dry cell identification. Computational cells with water depth $H \neq 0$ but with $H<<$ have to be identified and treated. To identify dry cells, a common treatment is to use a wet/dry tolerance $\varepsilon_{w d}$. If $H$ in a cell is computed lower to $\varepsilon_{w d}$ this cell is considered dry, i.e $H=0, u=0$. The choice of $\varepsilon_{w d}$ is not necessarily trivial and its value maybe related to the type of problem simulated and the mesh size [17].

Conservation of the flow at rest with dry regions. A numerical scheme should correctly compute the flow at rest exact solution (16) and (17) regardless of including wet/dry transitions and as such satisfy the extended $C$-property [10]. If no modifications are made, the numerical schemes presented in the previous section is not well-balanced in this sense for adverse dry slopes (emerging topography). To deal with this problem we have to redefine the bed elevation at the emerging dry cell following $[7,8,16,35]$. The reason for redefining the bed elevation is to obtain an exact balance at the front between the bed slope and the hydrostatic terms 
for steady conditions and to avoid the appearance of spurious pressure forces. Considering the case where a wet/dry front exists between computational cells with reconstructed face values $L$ and $R$. This redefinition (reduction) of the bed elevation is formulated as:

$$
\Delta b= \begin{cases}H^{L}, & \text { if } H^{L}>\varepsilon_{w d} \text { and } H^{R} \leq \varepsilon_{w d} \text { and } b^{R}<\left(b^{R}-b^{L}\right), \\ \left(b^{L}-b^{R}\right), & \text { otherwise. }\end{cases}
$$

in the calculation of $\widetilde{\mathbf{S}_{\mathbf{b}}}$ in equations (29). In case that this treatment is not applied the water would climb over adverse bed slopes and the front will be wrongly diffused [7, 8, 10, 16, 35]. We note here that, the above redefinition of the bed elevation is not an actual reduction of the bed elevation value but is only applied in the calculation of $\widetilde{\mathbf{S}_{\mathbf{b}}}$ in (29). The numerical treatment is similar if $R$ is the wet side and $L$ is identified as dry.

Flow in motion over adverse slopes. With the above redefinition of the discretized topography one can treat situations of emerging topography for a flow at rest. However, further modifications have to be made for a flow in motion. At interfaces between a wet/dry front, we impose, additional to the above bed redefinition, the following temporary condition for the computation in $(23)$, following $[10,16,35]$,

$$
\text { if }\left[H^{L}>\varepsilon_{w d} \text { and } H^{R} \leq \varepsilon_{w d} \text { and } H^{L}<\left(b^{R}-b^{L}\right)\right] \text { then } u^{L}=u^{R}=0 .
$$

We note that, the actual velocity is not set to zero in the wet cell, but only assumed as such at the face when computing the numerical flux and topography source term contributions. As such, the solution at each wet/dry face is considered to be that corresponding to an emerging bottom situation for a water at rest and then, to compute the time evolved solution, the numerical fluxes and sources corresponding to this approximate situation at the face are used. In this way, the wet/dry front is only allowed to advance when the water gradient in the wet cell is larger than the bottom gradient between cells. In addition, this numerical treatment avoids the appearance of negative values for $H$ as well as un-physical overtopping $[10,11,16]$.

Depth positivity and mass conservation. In cases where the bed elevation of a dry cell is less to that of a wet cell (downhill slope) the flow will continue to flood the dry cell and there is no need to modify the bed slope. However, when the bed slope is steep, there is a possibility that more water, than is actually contained in the wet cell, could be computed as flowing into the dry cell, causing the water depth in the wet cell to become negative and the scheme to become unstable. In the present work and following [7] and [17], if this is the case, the cells with negative depth after one time step are identified and their water depth values and velocity components are set to zero. This adds a very small volume of water to the system. Then, the same volume of water is subtracted from all the wet cells (in the entire computational domain) in order to maintain mass conservation. This volume of subtracted mass is very small and as it is uniformly distributed to all the wet cells in the entire computational domain, an even smaller fraction of that mass volume is actually subtracted from each wet cell. Consequently, the possibility of numerically drying a wet cell during that procedure is almost non existent. The same procedure is adopted for those cells that have been identified as almost dry (by $\varepsilon_{w d}$ ), and their water depth was set to zero. Mass conservation has been monitored for the test cases considered in this work and the wet-dry treatment presented here is found to ensure absolute mass conservation.

We emphasize here that, while the above treatment steps have been proven successful for the NSWE, it is the first time that have been incorporated to the numerical solution of dispersive models. 


\subsection{FD discretizations of the dispersion terms}

In both models the dispersion terms contain spatial derivatives of up to third order. According to [49] a fourth-order accurate treatment of the first-order derivatives is required so that the truncation errors in the numerical schemes are smaller than the dispersion terms present in the models. As such, we discretize them using fourth-order central FD approximations for first order derivatives, third order central difference approximations for third order derivatives and second order for second order derivatives.

In Nwogu's formulation with (8), (9) and (10) and in order to discretize the term $\psi_{C}$ in the mass and momentum equations, we consider the cell average of this term as

$$
\begin{aligned}
\left(\psi_{c}\right)_{i}=\frac{1}{\Delta x} \int_{C_{i}} \psi_{c}= & \frac{1}{\Delta x}\left\{\left[\left(\frac{z_{a i+1 / 2}^{2}}{2}-\frac{d_{i+1 / 2}^{2}}{6}\right) d_{i+1 / 2}\left(u_{i+1 / 2}\right)_{x x}+\left(z_{a i+1 / 2}+\frac{d_{i+1 / 2}}{2}\right) d_{i+1 / 2}\left(d_{i+1 / 2} u_{i+1 / 2}\right)_{x x}\right]\right. \\
& \left.-\left[\left(\frac{z_{a i-1 / 2}^{2}}{2}-\frac{d_{i-1 / 2}^{2}}{6}\right) d_{i-1 / 2}\left(u_{i-1 / 2}\right)_{x x}+\left(z_{a i-1 / 2}+\frac{d_{i-1 / 2}}{2}\right) d_{i-1 / 2}\left(d_{i-1 / 2} u_{i-1 / 2}\right)_{x x}\right]\right\}
\end{aligned}
$$

Then, by substituting cell averaged values into the Taylor series we can express a cell averaged value with values defined at cell interfaces [26, 28] and through manipulation of Taylor series expansions we can derive the following disretizations, used in (33),

$$
\phi_{i+1 / 2}=\frac{7\left(\phi_{i+1}+\phi_{i}\right)-\left(\phi_{i+2}-\phi_{i-1}\right)}{12}+O\left(\Delta x^{4}\right), \quad\left(\phi_{i+1 / 2}\right)_{x x}=\frac{\left(\phi_{i+2}+\phi_{i-1}\right)-\left(\phi_{i+1}-\phi_{i}\right)}{2 \Delta x^{2}}+O\left(\Delta x^{2}\right) .
$$

For the momentum dispersion term $\psi_{M}$ in (9) we discretize the second order derivative using

$$
\left(u_{i}\right)_{x x}=\frac{u_{i-1}-2 u_{i}+u_{i+1}}{\Delta x^{2}}+O\left(\Delta x^{2}\right)
$$

thus $\psi_{M}$ is then given by

$$
\left(\psi_{M}\right)_{i}=\left(H_{i}\right)_{t} z_{a i}\left(\frac{z_{a i}}{2} \frac{u_{i-1}-2 u_{i}+u_{i+1}}{\Delta x^{2}}+\frac{d_{i-1} u_{i-1}-2 d_{i} u_{i}+d_{i+1} u_{i+1}}{\Delta x^{2}}\right) .
$$

with term $\left(H_{i}\right)_{t}$ explicitly obtained from the mass equation in terms of spatial derivatives only.

For the MS equations we discretize the term $\psi,(14)$, of the momentum equation, using

$$
\left(\phi_{i}\right)_{x}=\frac{\phi_{i-2}-8 \phi_{i-1}+8 \phi_{i+1}-\phi_{i+2}}{12 \Delta x}+O\left(\Delta x^{2}\right), \quad\left(\phi_{i}\right)_{x x x}=\frac{\phi_{i+2}-2 \phi_{i+1}+2 \phi_{i-1}-\phi_{i-2}}{2 \Delta x^{3}}+O\left(\Delta x^{3}\right) .
$$

Applying (36) and (34) in equation (14) we get the discrete dispersion term:

$$
\psi_{i}=-\frac{B g d_{i}^{3}}{\Delta x^{3}}\left[\eta_{i+2}-2 \eta_{i+1}+2 \eta_{i-1}-\phi_{i-2}\right]-\frac{B g d_{i}^{2}}{6 \Delta x^{3}}\left[\left(d_{i-2}-8 d_{i-1}+8 d_{i+1}-d_{i+2}\right)\left(\eta_{i-1}-2 \eta_{i}+\eta_{i+1}\right)\right]
$$

\subsection{Time integration}

Applying the fourth-order predictor-corrector method proposed in [49], time integration is achieved in two stages as follows,

1. Predictor stage (Adams-Basforth method) 


$$
\begin{array}{r}
\mathbf{U}_{i}^{p}=\mathbf{U}_{i}^{n}+\frac{\Delta t}{12 \Delta x}\left[23\left(-\Delta \mathbf{F}_{i}^{n}+\Delta \mathbf{S}_{\mathbf{b}_{i}}^{n}+\Delta x \mathbf{S}_{\mathbf{d} i}^{n}+\Delta x \mathbf{S}_{\mathbf{f} i}^{n}\right)-\right. \\
-5\left(-\Delta \mathbf{F}_{i}^{n-1}+\Delta \mathbf{S}_{\mathbf{b}_{i}}^{n-1}+\Delta x \mathbf{S}_{\mathbf{d} i}^{n-1}+\Delta x \mathbf{S}_{\mathbf{f} i}^{n-1}\right) \\
\left.+5\left(-\Delta \mathbf{F}_{i}^{n-2}+\Delta \mathbf{S}_{\mathbf{b}_{i}}^{n-2}+\Delta x \mathbf{S}_{\mathbf{d} i}^{n-2}+\Delta x \mathbf{S}_{\mathbf{f} i}^{n-2}\right)\right]
\end{array}
$$

2. Corrector stage (Adams-Moulton method)

$$
\begin{aligned}
\mathbf{U}_{i}^{n+1}= & \mathbf{U}_{i}^{n}+\frac{\Delta t}{24 \Delta x}\left[9\left(-\Delta \mathbf{F}_{i}^{p}+\Delta \mathbf{S}_{\mathbf{b}_{i}}^{p}+\Delta x \mathbf{S}_{\mathbf{d} i}^{p}+\Delta x \mathbf{S}_{\mathbf{f} i}^{p}\right)+19\left(-\Delta \mathbf{F}_{i}^{n}+\Delta \mathbf{S}_{\mathbf{b}_{i}}^{n}+\Delta x \mathbf{S}_{\mathbf{d} i}^{n}+\Delta x \mathbf{S}_{\mathbf{f} i}^{n}\right)\right. \\
& \left.-5\left(-\Delta \mathbf{F}_{i}^{n-1}+\Delta \mathbf{S}_{\mathbf{b}_{i}}^{n-1}+\Delta x \mathbf{S}_{\mathbf{d} i}^{n-1}+\Delta x \mathbf{S}_{\mathbf{f} i}^{n-1}\right)+\left(-\Delta \mathbf{F}_{i}^{n-2}+\Delta \mathbf{S}_{\mathbf{b}_{i}}^{n-2}+\Delta x \mathbf{S}_{\mathbf{d} i}^{n-2}+\Delta x \mathbf{S}_{\mathbf{f} i}^{n-2}\right)\right]
\end{aligned}
$$

where $\Delta \mathbf{F}_{i}$ and $\Delta \mathbf{S}_{\mathbf{b} i}$ are respectively the flux and bed source variations from (24), $\mathbf{S}_{\mathbf{d} i}$ is the discretization of the dispersion terms while $\mathbf{S}_{\mathbf{f} i}$ is the friction term from (15).

In the predictor as well as in the corrector step we obtain values for $H_{i}$ and $P_{i}^{\star}$ in (8) for Nwogu's model and values for $H_{i}$ and $\tilde{P}_{i}^{\star}$ in (13) for the MS model, from which the velocity value $u_{i}$ should be evaluated. For Nwogu's formulation, using (34) to discretize the spatial derivatives in (8) and after some factorization, the velocity value $u$ is obtained by solving the following tridiagonal linear system of equations:

$$
\alpha_{i} u_{i-1}+\beta_{i} u_{i}+\gamma_{i} u_{i-1}=P_{i}^{\star}, \quad i=1, \ldots, n
$$

where

$$
\alpha_{i}=H_{i} z_{a i}\left(\frac{z_{a i}}{2 \Delta x^{2}}+\frac{d_{i-1}}{\Delta x^{2}}\right), \beta_{i}=H_{i}\left(1-\frac{z_{a i}^{2}}{\Delta x^{2}}-\frac{2 z_{a i} d_{i}}{\Delta x^{2}}\right) \text { and } \gamma_{i}=H_{i} z_{a i}\left(\frac{z_{a i}}{2 \Delta x^{2}}+\frac{d_{i+1}}{\Delta x^{2}}\right)
$$

An efficient Thomas algorithm was used to solve the system for obtaining the velocity $u_{i}^{p}$ in the predictor step and $u_{i}^{n+1}$ in the corrector one, from the respective variables $P_{i}^{\star}$.

The velocity function $\tilde{P}_{i}^{\star}$ in (13) for the MS equations requires the approximation of first and second order derivatives. Using approximations from (34) for $\left(H_{i} u_{i}\right)_{x x}$, from (36) for $\left(d_{i}\right)_{x}$ and the central difference $\left(H_{i} u_{i}\right)_{x}=\left(H_{i+1} u_{i+1}-H_{i-1} u_{i-1}\right) / 2 \Delta x$, equation (13) results again in a tridiagonal system in the form of (40) with coefficients, see also $[21,40,45]$,

$$
\tilde{\alpha}_{i}=\left[-k_{1}+\frac{k_{2}}{72}\right], \quad \tilde{\beta}_{i}=1+2 k_{1}, \quad \tilde{\gamma}_{i}=-\left[k_{1}+\frac{k_{2}}{72}\right] .
$$

in which

$$
k_{1}=\left(B+\frac{1}{3}\right)\left(\frac{d_{i}^{2}}{\Delta x^{2}}\right) \text { and } k_{2}=\frac{d_{i}}{\Delta x^{2}}\left(d_{i-2}-8 d_{i-1}+8 d_{i+1}-d_{i+2}\right) .
$$

As suggested in [49] the corrector step is iterated until the relative error between two successive results reaches a prescribed tolerance. This error is computed for each of the variables $H$ and $u$ and is defined as

$$
e=\frac{\sum_{i}\left|v_{i}^{n+1}-\tilde{v}_{i}^{n+1}\right|}{\left|\sum_{i} v_{i}^{n+1}\right|}
$$


where $v$ denotes any of the variables and $\tilde{v}$ denotes the previous estimate. In this work the values computed in the corrector step are considered to be the final next time step $(n+1)$ values when $e<10^{-4}$.

The value of the time step, $\Delta t$, that is used in the time integration in (38) and (39) is dependent upon the CFL condition, that is

$$
\Delta t=C_{r} \min _{i}\left(\frac{\Delta x}{\left|u_{i}\right|+\sqrt{g H_{i}}}\right)
$$

where $C_{r}$ is the Courant number between zero and one.

\subsection{Boundary conditions}

To completely define the differential problems, boundary conditions need to be introduced. Two types of boundary conditions have been considered in this study: outflow and solid (reflective) wall. In a computational domain the reconstructed values, for the fourth-order MUSCL scheme, on the first and last cell are computed using neighboring ghost cells. More precisely, at each boundary three ghost nodes are needed, the values of which are determined by application of boundary conditions.

For an outflow boundary condition the values of $H$ and $u$ at the ghost cells are computed from the interior by taking odd and even extrapolation [29, 40]. In addition, all energy propagating towards the boundary should pass through so that no unphysical waves are reflected back into the solution domain due to dispersive effects. In order to dissipate the energy of reflective waves perfectly, a sponge layer, [45], is defined in front of this kind of boundaries where the velocity is gradually damped near the boundaries. If the dispersive terms are ignored in the models, i.e. when solving the NSWE, there is usually no need to activate the sponge layer and classical numerical boundary conditions for the NSWE can be easily applied. Solid walls are treated using an odd and even extrapolation for $H$ while for the velocity $u_{-g}$ at a ghost cells is set as $u_{-g}=-u_{g}$ from the interior. These conditions reflect incident waves on the boundaries conservatively.

\section{Wave breaking treatment}

As a wave's amplitude increases and reaches a critical level the wave crest steepens, the front of the wave becomes vertical and then the crest of the wave overturns. It should be noted that, wave breaking is a complex phenomenon, with substantial air entrainment, turbulence and energy dissipation. At this point models of the Boussinesq-type are unable to describe the physical procedure since the FD discretization of the dispersive terms causes the generation of spurious oscillations if steep gradients are presented. Thus, a wave breaking mechanism has to be considered.

To ensure the stability of the simulations without resorting to any artificial filtering, smoothing to the solution or adding extra dissipative terms, a simple breaking mechanism, proposed in [45] and [46] for the MS equations, was applied and tested here also for Nwogu's model. The idea behind the proposed approach in [45] is that Boussinesq equations degenerate into NSWE as dispersive terms become negligible compared to nonlinear terms. When waves move towards a beach the water depth decreases, the dispersive effects becomes small and $\epsilon=A / d$ which represents the nonlinearity, grows. Using the idea that the transitions between non-breaking and breaking waves are similar to those between undular bores (which can be model by means of Boussinesq-type equations) and fully developed jumps (which can be modeled by the NSWE) and that the limiting condition for the appearance of an undular bore corresponds to a Froude number of 1.6, the value of $\epsilon$ was calculated to be the criterion to switch from Boussinesq equations to NSWE. Consequently, in each computational cell we check $\epsilon$, if $\epsilon \leq 0.8$ Boussinesq equations are solved, otherwise the dispersive terms are switched off and the NSWE are solved. Actually, this value is very close to that of the highest stable solitary wave which is for $A / d=0.78$ [51] and provides a similar breaking criterion to the one in [2] 
where a similar hybrid Boussinesq/NSWE model for the MS equations was presented. Thus, the model intrinsically captures the location and evolution of the breaking event. This is a one parameter approach which is not subject to calibration and avoids the necessity of introducing complex algorithms to track breaking wave fronts hence its complexity is limited compared to other breaking models e.g. eddy viscosity ones. Given its simplicity, the proposed model does not aim at describing the details of wave breaking dynamics at a local scale but at efficiently and accurately simulating its global effects as well as, providing stable and robust results. We note here that, application and comparison of other breaking models, especially those using the popular eddy viscosity approach, see for example [25, 26, 39, 51], although not in the scope of this work, may be beneficial for the current models and will be presented elsewhere.

\section{Numerical tests and results}

\subsection{The Carrier and Greenspan (1958) solutions}

We first check the validity of the proposed well-balanced discretization and wet/dry front treatment by focusing on moving shoreline problems for waves running up and down uniform sloping beaches. These problems have analytical solutions which were derived by Carrier and Greenspan [9] by making use of the NSWE. These classical tests have been used frequently for assessing the quality of various shoreline boundary condition techniques used in the NSWE, see for example $[4,6,23,34]$. Thus, for consistency the dispersive $\left(\mu^{2}\right)$ terms are usually ignored in the numerical simulations of these problems, see for example $[13,31,46]$, or considered to be very small [22]. In the current presentation, and only for these test cases, we chose to ignore the dispersive terms. Details on initial and boundary conditions, as well as the analytical solutions, can be found in the original work of Carrier and Greenspan [9] and in [6, 34].

Non-dimensional variables are defined to compare with the analytical solutions as follows

$$
x^{*}=x / l, \quad \eta^{*}=\eta /(\alpha l), \quad u^{*}=u / \sqrt{g \alpha l} \quad t^{*}=t / \sqrt{l /(\alpha g)}
$$

where $l$ is the characteristic length scale an $\alpha$ the beach slope.

The Carrier and Greenspan periodic wave solution. For the first case a monochromatic wave is let runup and rundown on a plane beach with $\alpha=1 / 30$. The solution represents the motion of a periodic wave of a dimensionless amplitude $A^{*}=0.6$ and frequency $\omega^{*}=1$ traveling shoreward and being reflected out to sea generating a standing wave on the beach with $A^{*} / 4$ maximum vertical excursion of the shoreline. The characteristic length scale $l=20 \mathrm{~m}$. The computational parameters used where $\Delta x=0.00625 \mathrm{~m}, C_{r}=0.45$ and $\varepsilon_{w d}=5 \cdot 10^{-6} \mathrm{~m}$. The small value of $\Delta x$ has been chosen as to provide a high resolution at the shoreline, see for example [46]. Fig. 1 presents comparisons between the numerical free surface and the analytical one, at different times $t^{*} \in\left[2 T, 2 T^{*}+T^{*} / 2\right]$ where $T^{*}$ is the dimensionless period of the oscillations. The qualitative agreement is very good even after a few periods.

The numerical shoreline position $x_{s}$ was measured as the position of the last wet cell (i.e. where $H \geq \varepsilon_{w d}$ ) along the beach. Theoretically, the shoreline boundary is defined by the relations

$$
H\left(x_{s}, t\right)=0 \quad \text { and } \quad u_{s} \equiv u\left(x_{s}, t\right)=\frac{d x_{s}}{d t} .
$$

Inevitably, the numerical solution produced by the conservative FV scheme is an approximation to the above relations since the zero depth condition is never met [3]. Fig. 2 compares the computed and analytical time series of the shoreline position and velocity. Very good accuracy is shown for the vertical sinusoidal motion of the shoreline while for the non-sinusoidal variation of the shoreline velocity small discrepancies are 


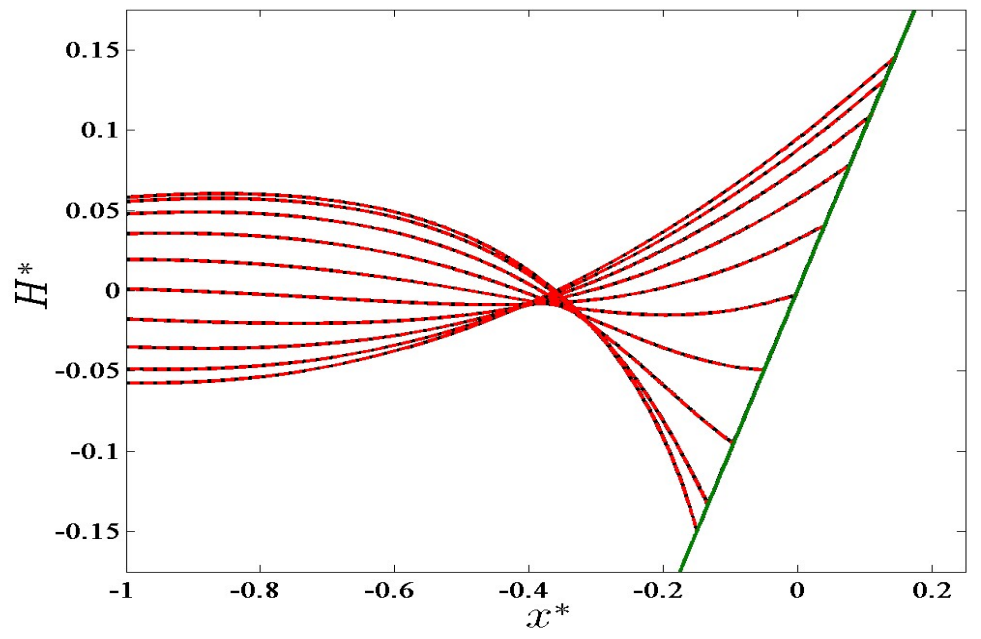

Figure 1: The Carrier and Greenspan periodic wave solution: comparison between analytical (solid line) and numerical (red dashed line) results for the free surface elevation at different times $t^{*} \in\left[2 T^{*}, 2 T^{*}+T^{*} / 2\right]$.

presented when the velocity is close to zero. The velocity results compare well with others in the literature, see for example $[22,46]$. It should be pointed here that the shoreline velocity computation is a difficult task and as such is not correctly captured by some runup models thus, in the literature only variations of the shoreline position are usually presented.
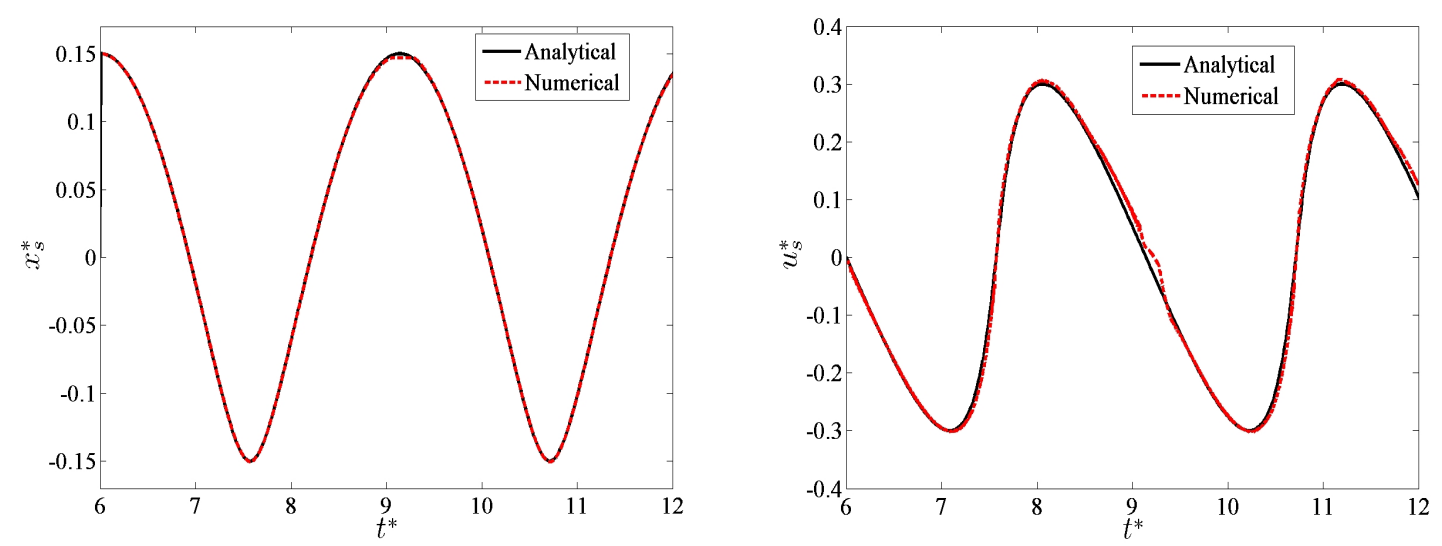

Figure 2: The Carrier and Greenspan periodic wave solution: comparison between analytical and numerical results for the shoreline position (left) and shoreline velocity (right)for $t^{*} \in\left[T^{*}, 2 T^{*}\right]$

The Carrier and Greenspan transient solution. In this case the initial water surface elevation is assumed to be depressed near the shoreline, the fluid is held motionless and then released at $t^{*}=0$. For this case $\alpha=1 / 50$ and $l=20 \mathrm{~m}$. In [9] a small parameter $e$ was used to characterize the surface elevation profile. For $e<0.23$ non-breaking waves are produced and the results presented here are for $e=0.1$. In the wave's 
evolution, the shoreline rises above the still water level of value $e$ and then the water surface elevation asymptotical settles back to it. As such, this test is particularly valuable in testing the ability of the model to compute nearly steady states. The computational parameters are the same as in the previous case.

Fig. 3 presents the comparisons between the numerical free surface and the analytical one, scaled with parameter $e$, at different times $t^{*}$. The numerical model provides qualitative excellent agreement with the analytic solution. The solution asymptotically reaches an almost steady state which is accurately predicted by the model verifying the extended $C$-property.

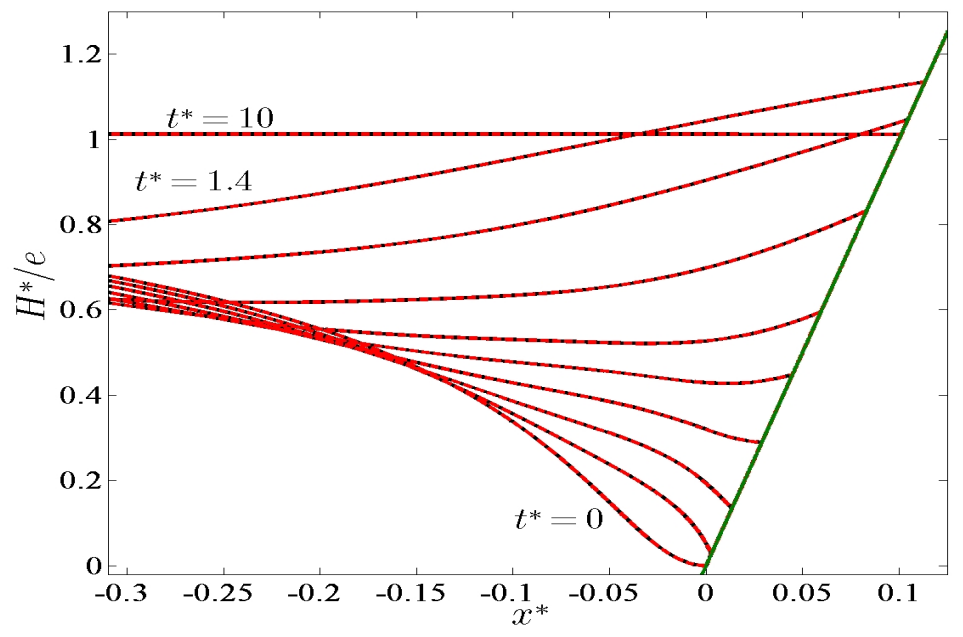

Figure 3: The Carrier and Greenspan transient solution: comparison between analytical (solid line) and numerical (red dashed line) results for the free surface elevation at different times $t^{*}$.

In Fig. 4 the computed and analytical time series of the shoreline position and velocity are presented. It can be observed that the shoreline position asymptotically settles to $e$ and the numerical model remains stable and with good accuracy for this slow convergence. On the other hand, the error on the shoreline velocity slightly increases when the velocity reaches very small values at rundown and exhibits a slightly oscillatory behavior. However, this error remains controlled and diminishing since the numerical solution fully recovers in a short time. This is an expected behavior since the method uses the threshold parameter $\varepsilon_{w d}$ to define the last wet cell. Hence, the intruduced error is mainly artificial since the velocity is calculated from the conserved variables as $u_{s}^{n+1}=(H u)_{s}^{n+1} / H_{s}^{n+1}$, with $(H u)_{s}^{n+1}$ being a few orders of magnitude less that $H_{s}^{n+1}$ and practically almost zero. Nevertheless, small spurious errors in the velocity or depth at wet/dry fronts which are below a certain level, e.g. $10^{-4}$, are not a concern in practical simulations as long as the solution remains stable for long time. Here, the numerical solution reaches stably the expected steady state.

\subsection{Solitary wave propagation}

This is one of the most standard tests of the stability and conservative properties of numerical schemes based on Boussinesq-type dispersive equations. A solitary wave maintains its shape, speed and amplitude as it travels down a flat $(b=0)$ frictionless channel due to an exact balance between the nonlinear terms that steepen the wave and the dispersion terms that flatten the wave. A numerical model must handle this balance. A combination of numerical errors from poorly balanced schemes and truncation of numerical approximations can lead to serious reductions in the wave height and celerity. 

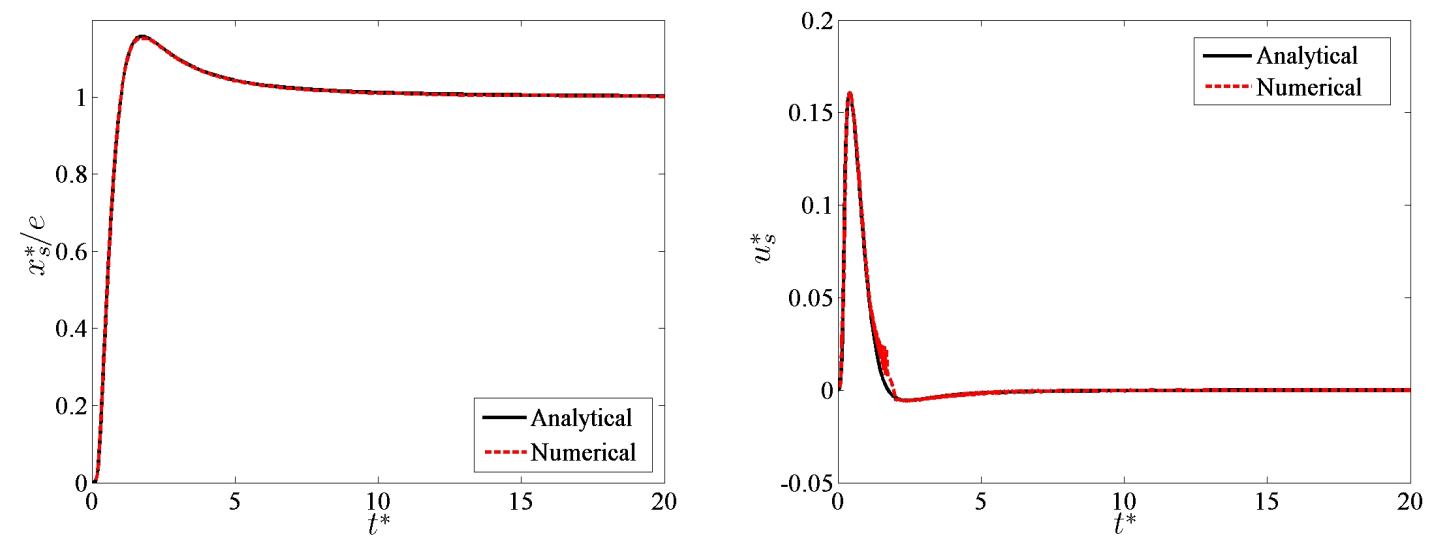

Figure 4: The Carrier and Greenspan transient solution: comparison between analytical and numerical results for the shoreline position (left) and shoreline velocity (right) for $t^{*} \in[0,20]$

A solitary wave of amplitude $A / d=0.1$ is considered for both formulations. The numerical wave channel is $500 \mathrm{~m}$ long with $d=0.45 \mathrm{~m}$. The initial wave surface elevation $\eta$ and velocity of $u$ can be found in [49] for Nwogu's equations and in [45] for the MS equations. The initial position of the solitary wave is at $x=0 \mathrm{~m}$. We note here that, the given initial conditions (and analytical solutions) are only asymptotically equivalent to the solutions of the models being solved numerically, so the wave being input in the numerical models does not correspond exactly to solitary waveforms predicted by the models. As a result, the initial waves undergo an evolution at the beginning of the computation to adjust the free surface to a steady profile [45, 49]. Fig. 5 shows the surface profile for the first wave for Nwogu's and MS conservative formulations respectively at times $t=0,50,100,150,200 s$ using $\Delta x=0.1 \mathrm{~m}$ and $C_{r}=0.4$. Nwogu's equations in conservation form produces a slightly higher wave that propagates with a constant shape and amplitude. In the MS formulation a train of small waves is formed which is soon left behind. Such transformation is attributed to the low-order approximation of the velocity, given to the model as initial condition. After some time the wave's shape is stabilized and reaches the numerical permanent form solitary wave solution, which propagates constantly. In
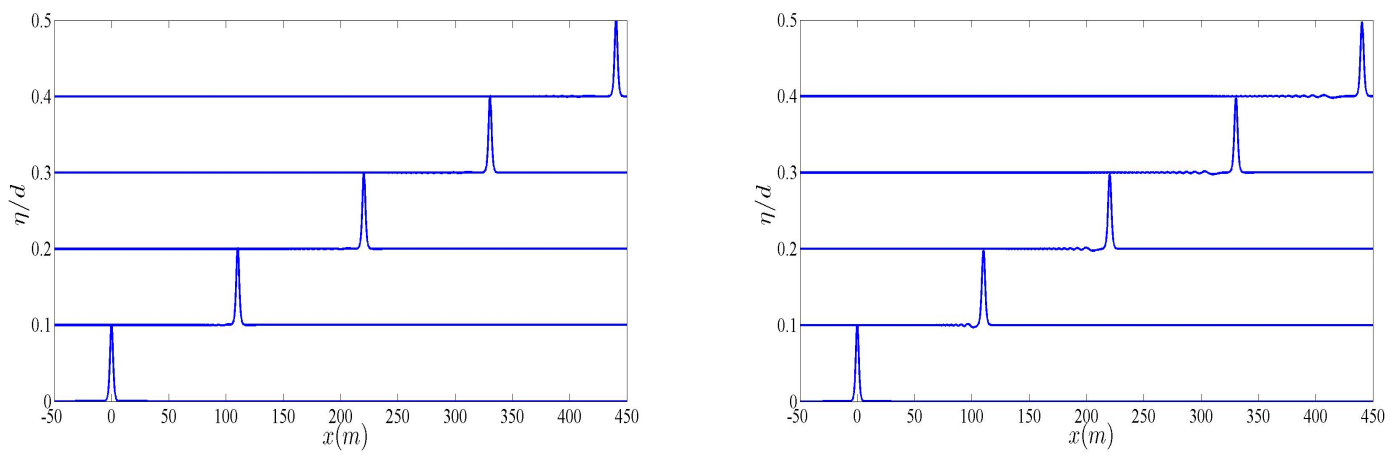

Figure 5: Solitary wave propagation $(A / d=0.1)$ at times $t=0,50,100,150,200$ s for Nwogu's (left) and MS (right) formulations.

Fig. 6 the solitary wave forms are compared to the analytical solution at two widely separated instances in time. The two waves are translated by a distance predicted through an analytical phase speed $C$ where it can 
be seen that the numerical wave shapes and speeds are in very close agreement with the analytical solutions.
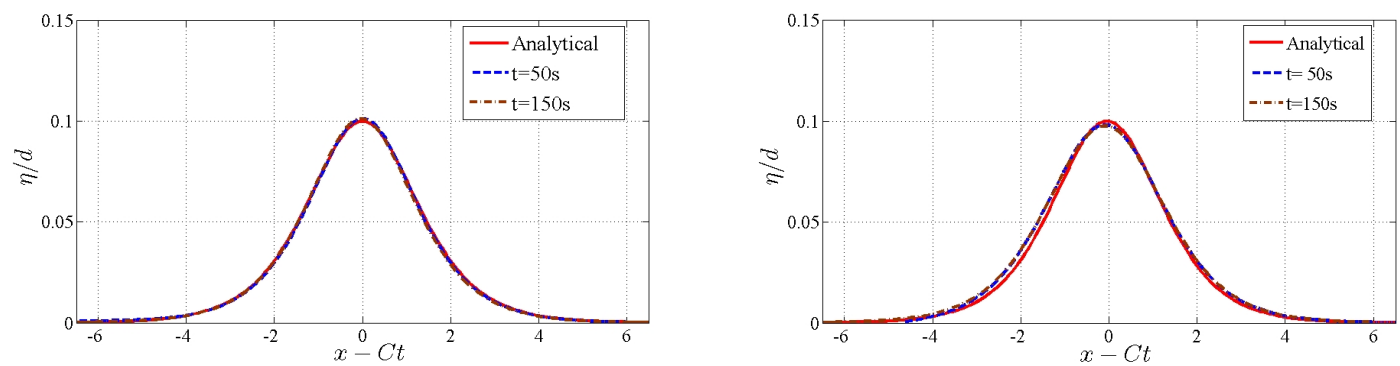

Figure 6: The solitary wave's $(A / d=0.1)$ shape at $t=50 \mathrm{~s}$ and $t=150 \mathrm{~s}$ for Nwogu's (left) and MS (right) formulations.

\subsection{Head on collision of two solitary waves}

The counter-propagation of two symmetric solitary waves in a flat frictionless channel is tested here. Their collision presents additional challenges to the models by the sudden change of nonlinear and dispersion characteristics. This head-on collision is characterized by the change of shape, along with a small phase-shift of the waves as a consequence of the nonlinearity and dispersion. In a $300 \mathrm{~m}$ long channel with $d=1 \mathrm{~m}$ two solitary waves with an equal initial hight of $A / d=0.3$ are placed in positions $x=35 \mathrm{~m}$ and $x=265 \mathrm{~m}$ and start to propagate in the opposite directions. This higher values of $A / d$ corresponds to stronger nonlinearity in the incident waves, when compared to the previous test case, since both models which are based on equations derived under the assumption that terms of $O\left(\epsilon^{2}, \epsilon \mu^{2}, \mu^{4}\right)$ can be neglected. The initial wave surface elevation and velocity can be found in [49] and [45], similar to the previous test case. The computational parameters used were $\Delta x=0.1 \mathrm{~m}$ and $C_{r}=0.4$.

Fig. 7 shows the surface profiles at times $t \sqrt{g / d}=0,101.2$ and 200. Similar to the previous test case and due to the incompatibility between the analytical and numerical solutions, the numerical waves initially undergo an evolution and two slightly higher solitary waves are formed which then propagate with a constant amplitude $(A / d=0.3135$ for Nwogu's equations and $A / d=0.295$ for the MS ones) until collision, along with small dispersive tails which are left behind. At $t \sqrt{g / d}=101.2$ the wave gets its highest pick, $A / d=0.66$ for Nwogu's formulation and $A / d=0.61$ for the MS formulation. At $t \sqrt{g / d}=200$ the numerical wave profiles are very close to the initial solitary waveform. After the collision we observe that the numerical solution has a small phase shift compared to the analytic one. The dispersive tails are more intense for the MS model due to the discrepancy between the analytical solution given as input to the model and the solitary wave solution of the numerical model. We remark here that, nonlinear effects for both Nwogu's and the MS model can be reasonably accounted for up to $A / d \approx 0.3$.

\subsection{Solitary wave runup on a plane beach}

In [42] the runup, rundown and wave breaking of solitary waves with various nonlinearities, $\epsilon=A / d$, on a plane beach of slope 1:19.85 was studied both experimentally and theoretically. In this section we perform comparisons of numerical solutions for the two Boussinesq models and the NSWE (solved by the same numerical method) and experimental data from [42]. Two different cases are considered here. For the first case the incident wave hight is $A / d=0.04$ and for the second one $A / d=0.28$. According to [42] wave breaking occurred during rundown when $A / d>0.044$ and hence breaking did not occur for the first case (although it nearly broke on the rundown). The $A / d=0.28$ wave broke strongly during both the runup 

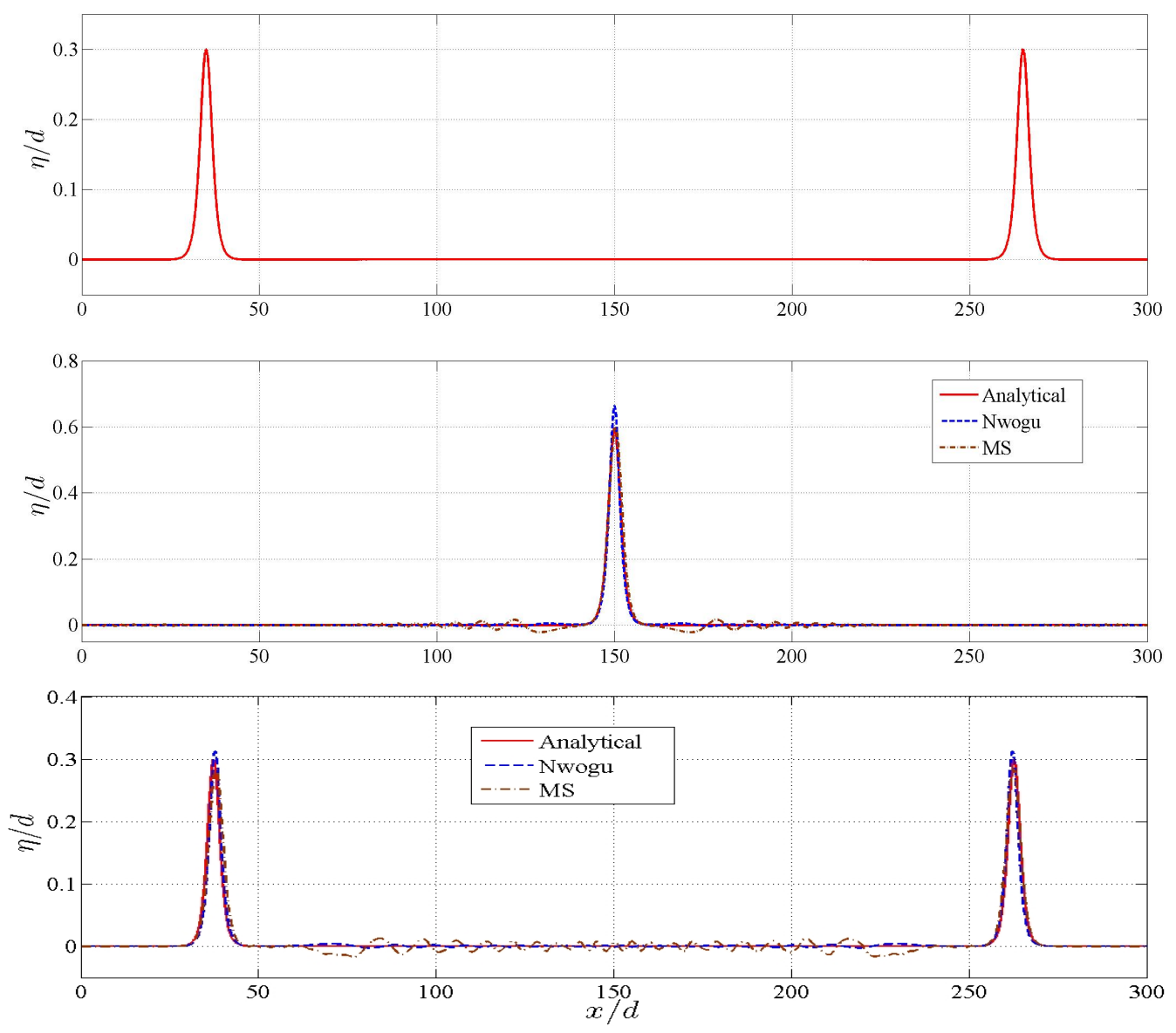

Figure 7: Surface profiles of solitary waves at times $t \sqrt{g / d}=0,101.2,200$ (from top to bottom) with $A / d=0.3$ propagating in opposite directions in a channel of constant depth.

and the rundown. We consider a computational domain for $x \in[-20,100 m]$ with $d=1 \mathrm{~m}$ with the initial solitary waves positioned at half the wave length from the beach toe. To obtain the correct initial conditions for the Boussinesq models, permanent-form solitary wave solutions of the desired height had to be found. Thus, similar to the tests in Sections 5.1 and 5.2, the initial condition form [49] for Nwogu's equations and the one from [45] for the MS were used to obtain these permanent forms. The maximum runup height, $\mathcal{R}$, was measured again as the maximum height of the last wet cell (i.e. where $H \geq \varepsilon_{w d}$ ). The computational parameters used were $\Delta x=0.1 \mathrm{~m}, C_{r}=0.3, \varepsilon_{w d}=5 \cdot 10^{-6}$ and $n_{m}=0.01$.

Fig. 8 presents the series of the surface profiles, along with experimental data, when $A / d=0.04$ for different non-dimensional times. Until time $t \sqrt{g / H}=32$ the wave approaches the shoreline and we observe that the NSWE slightly over-predict the solution while the numerical results of Nwogu's and MS give identical results for this non-breaking case. Observe that, due to the well-balanced wet/dry treatment applied in the shoreline no unphysical overtopping or movement appears in the solution. In time $t \sqrt{g / d}=32$ the runup process starts and after that the solutions of the three models are almost identical converging to the 
same solution. The experimental observation that the wave nearly broke during rundown can be seen at $t \sqrt{g / d}=62$ where all numerical solutions look similar to that of a hydraulic jump. The measured maximum runup in [42] for this problem was $\mathcal{R} \approx 0.156$ while for all computational models here was almost identical with a value $\mathcal{R} \approx 0.161$.
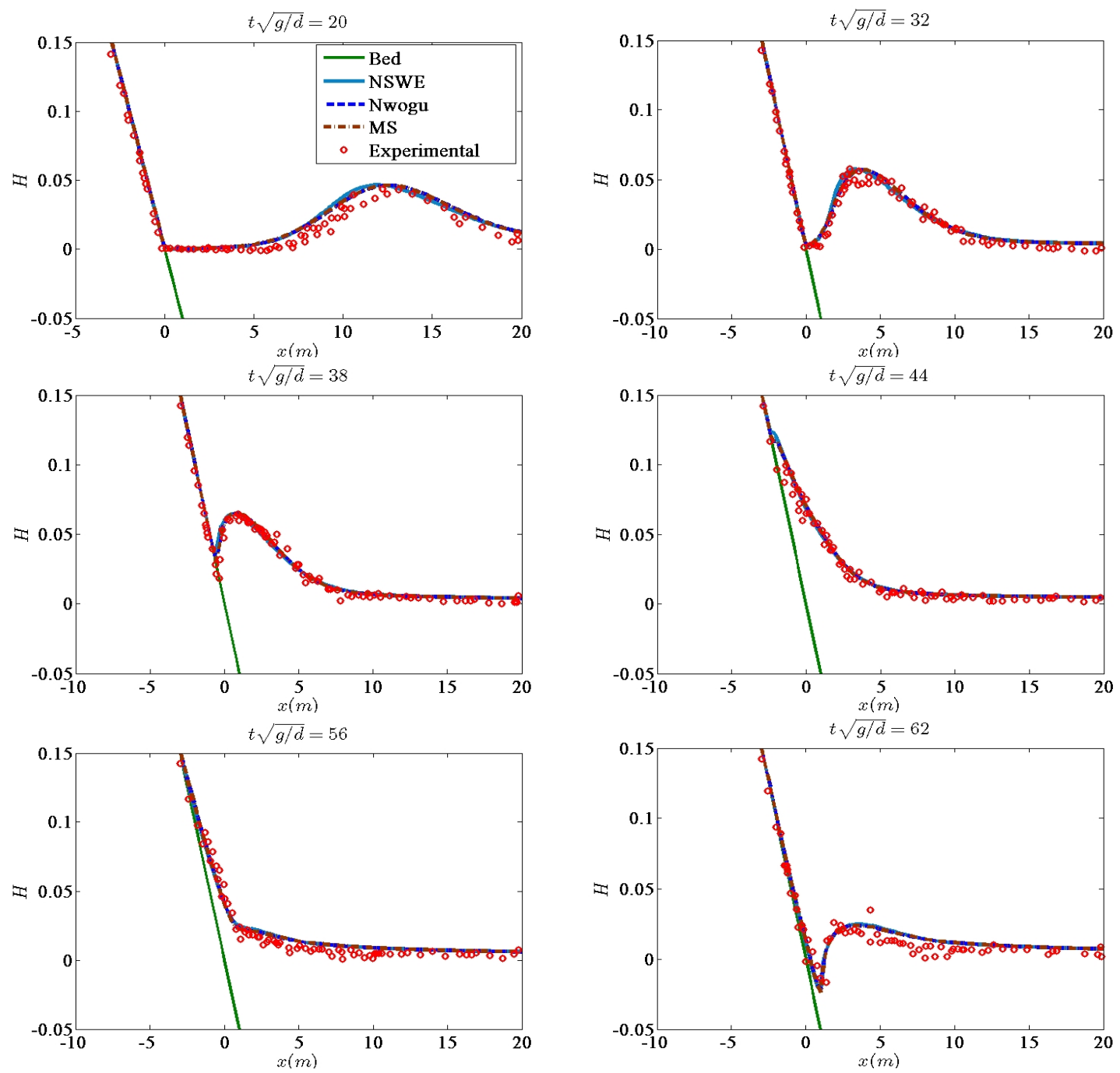

Figure 8: Free surface elevation of solitary wave runup on a plane beach for $A / d=0.04$

Fig. 9 presents the series of the surface profile when $A / d=0.28$. The breaking procedure starts around $t \sqrt{g / H}=15$ and the experimental wave breaks around $t \sqrt{g / H}=20$. As the wave shoals, it is clear that at the initial stages the NSWE under-predict the wave height and phase. The wave breaking is represented as a bore storing the water spilled from the breaking wave behind the front. The Boussinesq models, with the inclusion of the breaking treatment, approximate better the initial stages. The Boussinesq models reduce to NSWE during the breaking event, which approximate the turbulent breaking wave by a flow discontinuity 
hence, the computed front face becomes steeper. The actual wave is not discontinuous but contains air bubbles and turbulence. The slight mismatch between the experimental data and the numerical results are within acceptable limits, since the numerical formulations are not able to fully resolve the inherent flow processes to their full scale. Because of volume conservation in all models, the computed solution fully recovers until the water reaches the maximum run-up point around $t \sqrt{g / H}=45$. Since there is no single experimental value reported for $\mathcal{R}$ in [42] a theoretical interval was provided, i.e. $\mathcal{R} \in[0.42,0.53]$. The predicted value was $\mathcal{R} \approx 0.51$ for the Boussinesq models and $\mathcal{R} \approx 0.53$ for the NSWE. We note that, this value is strongly dependent on the friction value, [2, 31, 46], for this test case, but also on the $\varepsilon_{w d}$ value. In our tests for values of $\varepsilon_{w d} \leq 5 \cdot 10^{-6}$ the results didn't change, also for calculations with half and twice the current grid size. As the water recedes a breaking wave is created at $t \sqrt{g / H}=55$ near the still water level. In all numerical solutions this is approximated as a hydraulic jump with minor discrepancies with the laboratory data. Again, the computed results fully recover at later times.

\subsection{Solitary wave propagation over a reef}

The final experimental test case, initially presented in [39], is on solitary wave transformations over an idealized fringing reef that includes a fore reef slope and examines the capability of each model in handling simultaneously nonlinear dispersive waves together with wave breaking and bore propagation. The experiments were carried out at the O.H. Hinsdale Wave Research Laboratory at Oregon State University

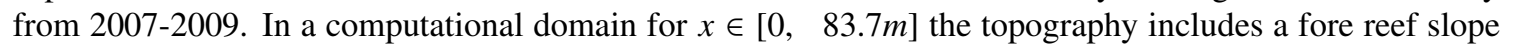
of $1 / 12$, a $0.2 \mathrm{~m}$ reef crest and a water depth $d=2.5 \mathrm{~m}$. The reef crest is then exposed by $6 \mathrm{~cm}$ and submerges the flat with $d=14 \mathrm{~cm}$. This test case involves a $0.75 \mathrm{~m}$ high solitary wave i.e. $A / d=0.3$. The computational parameters used were $\Delta x=0.1 \mathrm{~m}, C_{r}=0.3$ and $\varepsilon_{w d}=5 \cdot 10^{-6}$. Wall boundary conditions were placed at both ends of the computational domain. As suggested in [39], $n_{m}=0.012$ was used to define the roughness of the concrete surface in this challenging experiment. Experimental results for the free surface waves were recorded at 14 resistance wave gauges. We refer to [39] for the experimental set up and gauge locations.

Figs 10 and 11 compare the measured and computed wave profiles as the numerical solitary wave propagates. As the initially symmetric solitary wave shoals across the toe of the slope at $x=25.9 \mathrm{~m}$, it begins to skew to the front with the NSWE forming a vertically-faced propagating bore. Experimentally the wave began to break around $t=33.8 \mathrm{~s}$ developing a plunging breaker on the top of the reef crest that collapsed around $t=35 \mathrm{~s}$. All models are mimicking the breaker as a collapsing bore that slightly underestimates the wave height but conserved the total mass. By time $t=35.5 \mathrm{~s}$ the broken wave begins to travel down the back slope of the reef crest generating a supercritical flow that displaces the initially stagnant water, generating a hydraulic jump off the back reef and a downstream propagating bore fueled by the supercritical flow mass and momentum transfer. Around $t=45 \mathrm{~s}$ the momentum flux balances at the flow discontinuity and the hydraulic jump becomes stationary momentarily while the bore continues to propagate downstream. At subsequent time, the end wall reflects a bore back that by time $t=53.5 \mathrm{~s}$ has overtoped the reef crest generating a

hydraulic jump on the fore reef and a reflected bore at the back of the reef that travels again downstream. At this point, and as the water rushes down the fore reef, the flow transitions from flux to dispersion-dominated through the hydraulic jump. The created hydraulic jump at the fore reef propagates as a shock for the NSWE offshore due to the hyperbolic character of the equations, as can be seen in Figure 10 at times $t=53.5 \mathrm{~s}$ and $t=60.5 \mathrm{~s}$. On the other hand, the Boussinesq systems developed Airy type waves according to their dispersive characteristics for the intermediate depth offshore. By time $t=76.5 \mathrm{~s}$ waves have been reflected by both ends of the computational domain. Overall the computed results for the Boussinesq systems reproduce more accurately the wave transformations especially at later times and for the flow offshore from the 

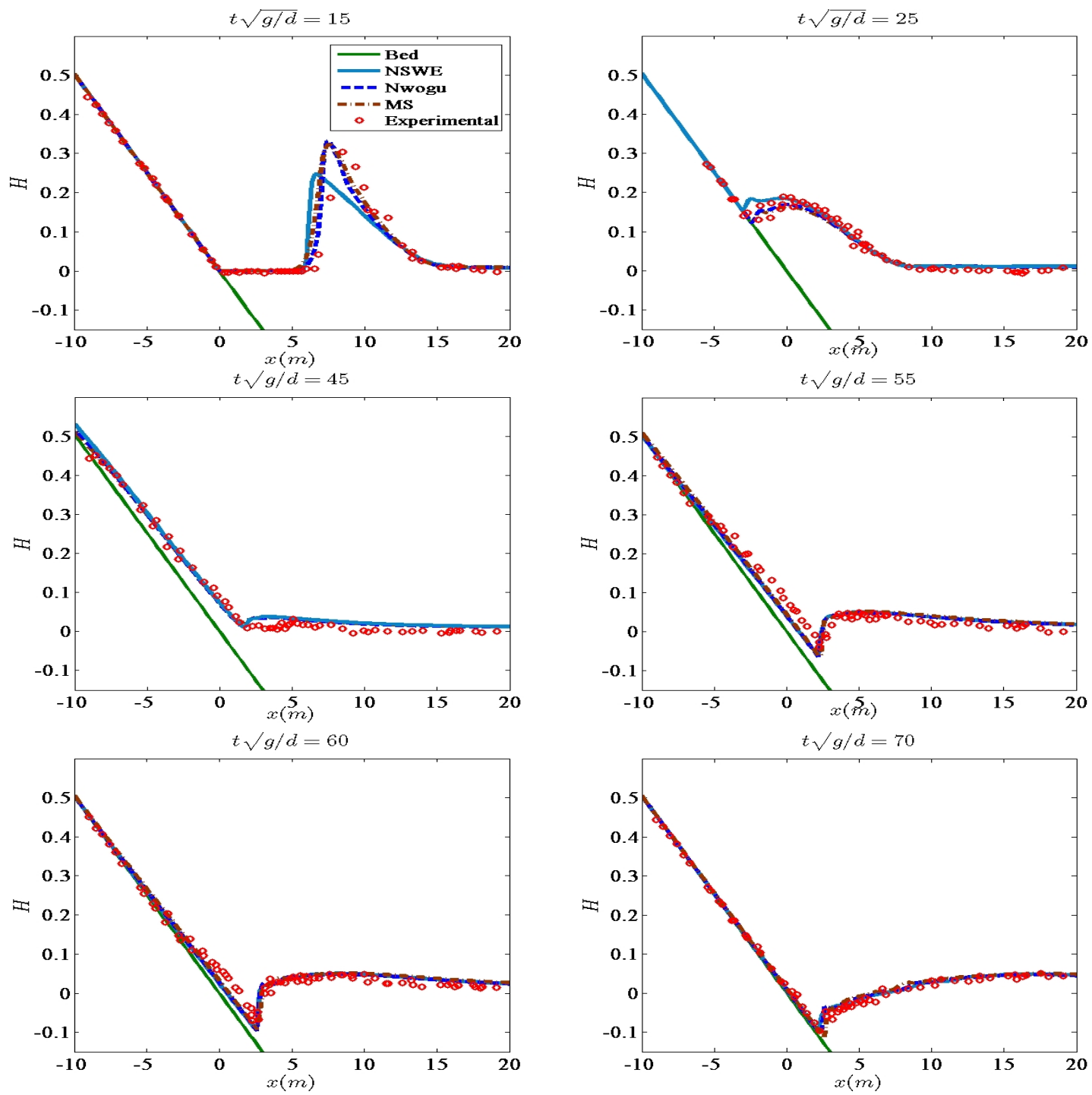

Figure 9: Free surface elevation of solitary wave runup on a plane beach for $A / d=0.28$

fore reef. The use of the breaking treatment is critical as the wave moves to the initially stagnant lagoon in order to capture the hydraulic jumps and reflected bores but then the results coincide for all models to this shallow region since the dispersive terms become almost zero and the Boussinesq models turn into the NSWE model as the breaking criterion is activated. It is important to note here that, the recognition and calculation of stationary and nearly stationary jumps on the flow profile, like the one that often forms during the draw down on a beach or the one formed from $t=35.5 \mathrm{~s}$ to $t=45 \mathrm{~s}$ constitutes a particularly challenging problem for Boussinesq-type models [39]. 

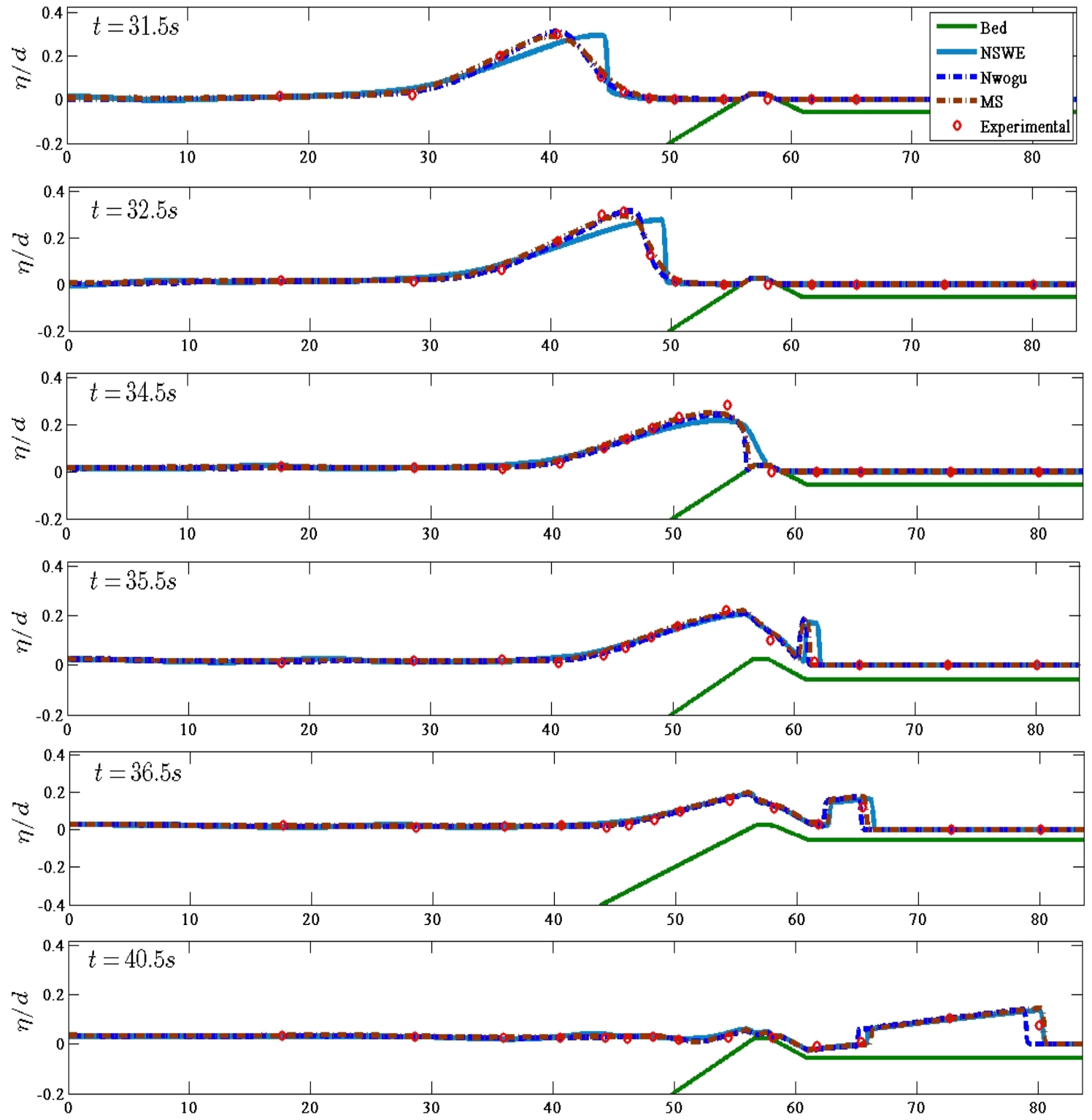

Figure 10: Evolution of normalized surface profiles and wave transformations over an exposed reef for $A / d=0.3$ and $1 / 12$ slope 

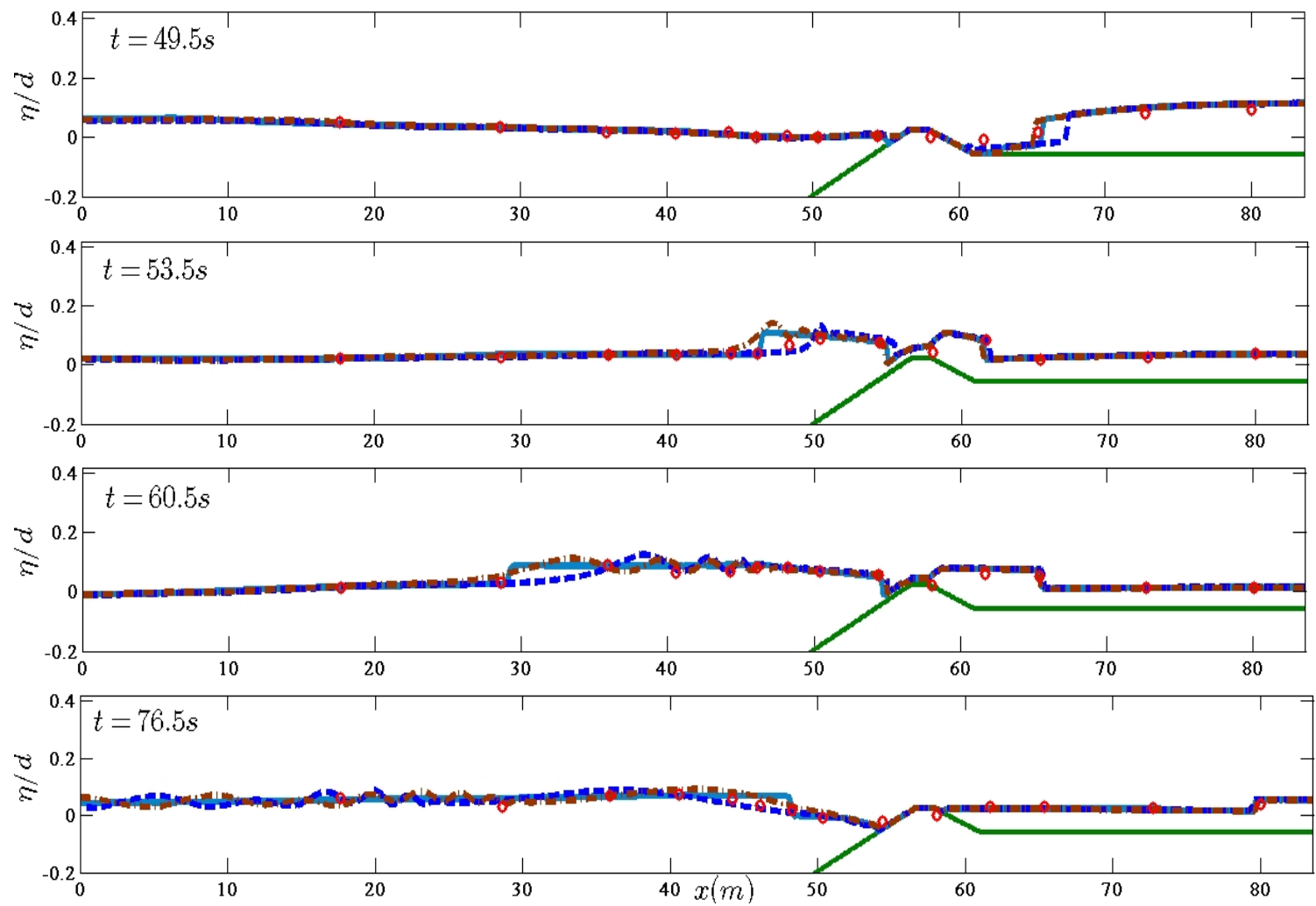

Figure 11: Evolution of normalized surface profiles and wave transformations over an exposed reef for $A / d=0.3$ and $1 / 12$ slope

Fig. 12 compares the computed and recorded surface elevation time series at specific wave gauges. The recorded data from the wave gauges at $x \leq 50.4 m$ shows the effect of the Airy type waves on the free surface. The hydraulic jump developed at the fore reef produced a train of waves over the increasing water depth and the resulting undulations were intensified as higher harmonics were released. As a matter of fact, wave gauges near the toe of the slope recorded highly dispersive waves of $k d>30$ [39]. The NSWE model totaly smooths the results with an additional phase shift. Nwogu's model expressed in conservative form managed to reproduce these highly dispersive waves with the correct phase and height strengths for this difficult problem and seems to compare in favor with the results presented in [39], although in Fig. 10 appears to give slower results, compared to the experimental data, at some time instances. For the MS model although it reproduced these high frequency oscillations for $x \leq 50.4 \mathrm{~m}$ a phase shift was observed resembling that of the NSWE model. The time series at $x=58.1 \mathrm{~m}$ present the initial and subsequently overtoppings at the reef crest and confirm the efficiency of the proposed wet/dry front treatment. The numerical models reproduced these overtoppings at the correct phase but slightly overestimated the height of the arrival waves. At the gauges located at $x=65.2 \mathrm{~m}$ and $72.6 \mathrm{~m}$ the arrival of the initial wave, the first reflected bore from the end wall, its subsequent reflection from the back reef as well as any subsequent reflections are almost correctly reproduced by the numerical models, verifying also the correct numerical boundary treatment, with Nwogu's model in conservative form being more accurate at later times. 

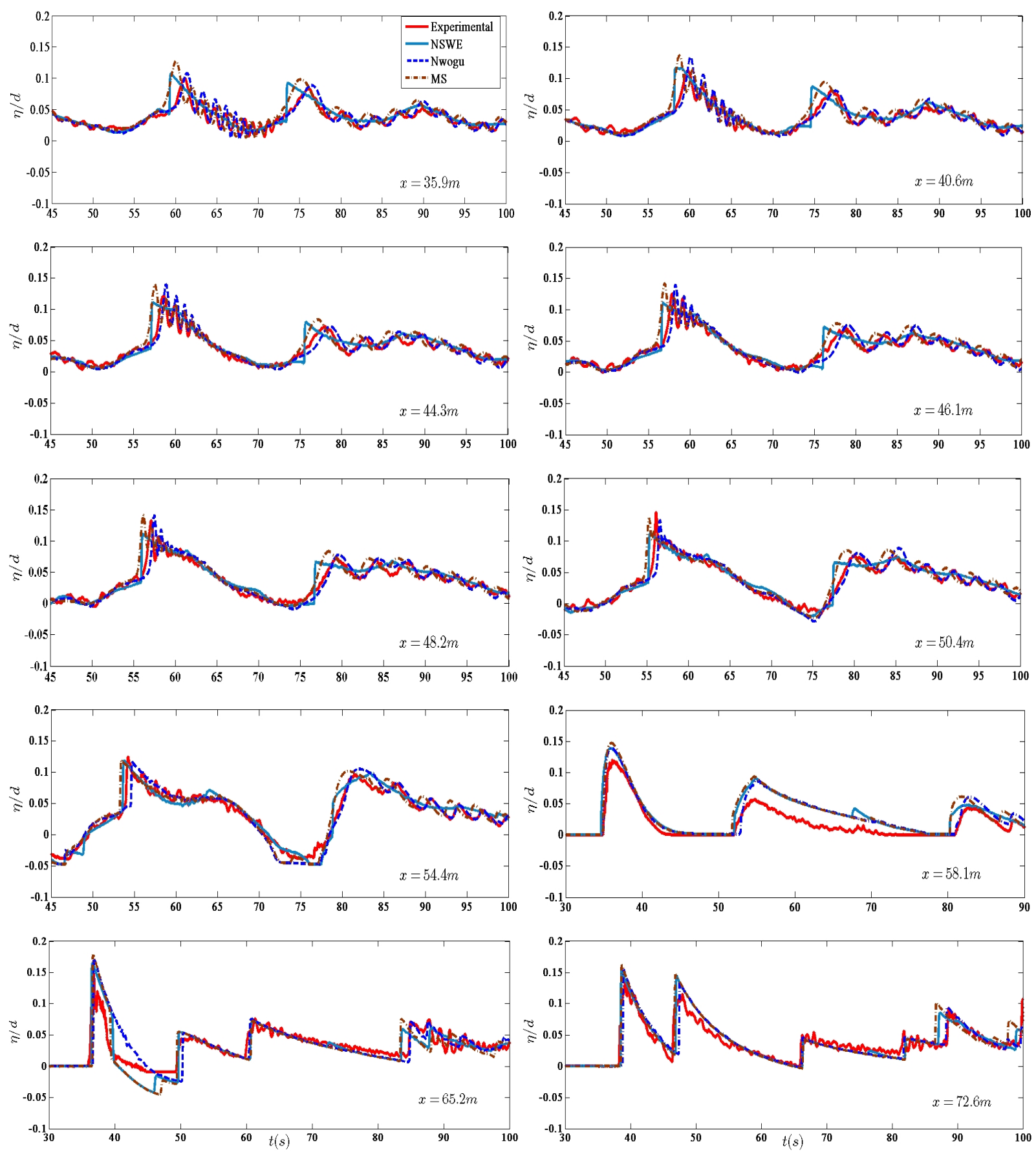

Figure 12: Time series of the normalized free surface at the wave gauges 


\section{Conclusions}

An alternative hybrid FV/FD numerical approach for the solution of the 1D extended Boussinesq-type equations of Nwogu and Madsen and Sørensen (MS) was presented. Both models were formulated in conservation law form incorporating the time-derivative component of the dispersion term into the vector of conserved variables and have an identical flux term as the nonlinear shallow water equations (NSWE). The application of a fourth-order conservative Godunov-type FV method for the evaluation of advective fluxes makes the proposed scheme shock-capturing. A specific and improved numerical treatment of natural topographies and the conservative computation of wet/dry fronts was also presented resulting in a wellbalanced scheme. As such, the treatment of the shoreline motion was extremely efficient, as the propagation of wet/dry interfaces was naturally represented resulting in accurate results for wave runup and rundown. For modeling breaking waves a previously introduced breaking criterion for the MS model was adopted and tested here for Nwogu's model as well. Utilizing this criterion results in a hybrid Boussinesq/NSWE modeling of breaking waves. The conservative formulation and proposed numerical scheme enhanced the capability and applicability of the Boussinesq models without altering significantly their dispersion characteristics. Special attention was paid to comparing both Boussinesq model to the NSWE for reproducing challenging experimental results. Results from this work confirm that, although the NSWE can be sufficient in some cases to predict maximum runup values and the general characteristics of propagating waves, the two Boussinesq-type numerical models provided considerable more accurate results for highly dispersive waves over increasing water depths, with Nwogu's model having a precedence over the MS one.

\section{Acknowledgement}

The authors wish to thank Dr. Volker Roeber for his helpful discussions during the course of this work, as well as for providing relevant experimental data.

\section{References}

[1] A. Bermudez, M. E. Vázquez-Cendón, Upwind methods for hyperbolic conservation laws with source terms, Computers and Fluids 23 (1994) 1049-1071.

[2] A. G. L. Borthwick, M. Ford, B. P. Weston, P. H. Taylor, P. K. Stansby, Solitary wave transformation, breaking and run-up at a beach, Maritime Engineering 159 (2006) 97-105.

[3] R. Briganti, N. Dodd, Shoreline motion in nonlinear shallow water coastal models, Coast. Eng. 56 (2009) 495.

[4] M. Brocchini, R. Bernetti, A. Mancinelli, G. Albertini, An efficient solver for nearshore flows based on the WAF method., Coastal Eng. 43 (2001) 105.

[5] M. Brocchini, N. Dodd, Nonlinear shallow water equations modeling for coastal engineering, J. Waterw. Port, Coastal, Ocean Eng. 134 (2008) 104.

[6] M. Brocchini, R. S. Svendsen, P. R. S., G. Bellotti, A comparison of two different types of shoreline boundary conditions, Comput. Methods Appl. Mech. Engrg 191 (2002) 4475-4496.

[7] P. Brufau, P. García-Navarro, M. E. Vázquez-Cendón, Zero mass error using unsteady wetting-drying coditions in shallow flows over dry irregular topography, Int. J. Numer. Meth. Fluids 45 (2004) 1047-1082.

[8] P. Brufau, M. E. Vázquez-Cendón, P. Gracía-Navarro, A numerical model for the flooding and drying of irregular domain, Int. J. Numer. Meth. Fluids 39 (2002) 247.

[9] G. F. Carrier, H. Greenspan, Water waves of finite amplitude on a sloping beach, J. Fluid Mech. 4 (1958) 97-109. 
[10] M. J. Castro, A. M. Ferreiro, J. A. García-Rodriguez, J. M. González-Vida, J. Macías, C. Parés, M. E. VázquezCendón, The numerical treatment of wet/dry fronts in shallow flows: Application to one-layer and two-layer systems, Mathematical and Computer Modelling 42 (2005) 419-439.

[11] M. J. Castro, J. M. González-Vida, C. Parés, Numerical treatment of wet/dry fronts in shallow flows with a modified Roe scheme, Math. Models and Meth. in Ap. Sc. 16 (2006) 897-931.

[12] R. Cienfuegos, E. Barthélemy, P. Bonneton, A fourth-order compact finite volume scheme for fully nonlinear and weakly dispersive Boussinesq-type equations. Part I: Model development and analysis, Int. J. Numer. Methods Fluids 51 (2006) 1217-1253.

[13] R. Cienfuegos, E. Barthélemy, P. Bonneton, A fourth-order compact finite volume scheme for fully nonlinear and weakly dispersive Boussinesq-type equations. Part II: Boundary conditions and validation, Int. J. Numer. Methods Fluids 53 (2007) 1423-1455.

[14] R. Cienfuegos, E. Barthélemy, P. Bonneton, Wave-breaking model for Boussinesq-type equations including roller effects in the mass conservation equation, J. Waterw., Port, Coast., Ocean Engrg. 136 (2010) 10-26.

[15] A. I. Delis, M. Kazolea, Finite volume simulations of waves formed by sliding masses, Comm. Num. Meth. Engng. 27 (2011) 732-757.

[16] A. I. Delis, M. Kazolea, N. A. Kampanis, A robust high resolution finite volume scheme for the simulation of long waves over complex domain, Int. J. Numer. Meth. Fluids 56 (2008) 419-452.

[17] A. I. Delis, I. K. Nikolos, M. Kazolea, Performance and comparison of cell-centered and node centered unstructured finite volume discretizations for shallow water free surface flows, Archives of Computational Methods in Engineering 18 (2011) 57-118.

[18] V. A. Dougalis, D. E. Mitsotakis, Theory and numerical analysis of Boussinesq systems: A review, in: N. A. Kampanis, V. A. Dougalis, J. A. Ekaterinaris (eds.), Effective Computational Methods in Wave Propagation, CRC Press, 2008.

[19] D. Dutykh, T. Katsaounis, D. E. Mitsotakis, Finite volume schemes for dispersive wave propagation and runup, Journal of Computational Physics 230 (2011) 3035-3061.

[20] K. S. Erduran, Further application of hybrid solution to another form of Boussinesq equations and comparisons, Int. J. Numer. Methods Fluids 53 (2007) 827-849.

[21] K. S. Erduran, S. Ilic, V. Kutija, Hybrid finite-volume finite-difference scheme for the solution of Boussinesq equations, Int. J. Numer. Methods Fluids 49 (2005) 1213-1232.

[22] D. R. Fuhrman, P. A. Madsen, Simulation of nonlinear wave run-up with a high-order Boussinesq model, Coast. Eng. 55 (2008) 139-154.

[23] M. E. Hubbard, N. Dodd, A 2D numerical model of wave runup and overtoping, Coastal Eng. 47 (2002) 1-26.

[24] M. E. Hubbard, P. García-Navarro, Flux difference splitting and the balancing of source terms and flux gradients, J. Comp. Phys. 165 (2000) 89-125.

[25] A. B. Kennedy, J. T. Chen, Q. Kirby, R. A. Dalrymple, Boussinesq modeling of wave transformation, breaking and runup. Part I: 1D., J. Waterw., Port, Coast., Ocean Engrg. 126 (2000) 39-47.

[26] D.-H. Kim, P. J. Lynett, S. A. Socolofsky, A depth-integrated model for weakly dispersive, turbulent, and rotational fluid flows, Ocean Modelling 27 (2009) 198-214.

[27] J. T. Kirby, Boussinesq models and applications to nearshore wave propagation, surfzone processes and waveinduced currents, in: V. C. Lakhan (ed.), Advances in Coastal Modeling, Volume 67, 2003.

[28] C. A. Lacor, S. A. Smirnov, M. Baelmans, A finite volume formulation of compact central schemes on arbitrary structured grids, Journal of Computational Physics 198 (2004) 535-566.

[29] R. J. LeVeque, Finite Volume Methods for Hyperbolic Problems, Cambridge University Press, 2002.

[30] Y. Li, F. Raichlen, Non-breaking and breaking solitary wave run-up, J. Fluid Mech. 456 (2002) 295-318. 
[31] P. J. Lynett, T. R. Wu, P. L. F. Liu, Modeling wave runup with depth integrated equations, Coastal Eng. 46 (2002) 98-107.

[32] P. A. Madsen, H. A. Schäffer, Higher-order Boussinesq-type equations for surface gravity waves: derivation and analysis, Philos. Trans. R. Soc. Lond. 356 (1998) 3123-3184.

[33] P. A. Madsen, O. R. Sørensen, A new form of the Boussinesq equations with improved linear dispersion characteristics. Part 2: A slowing varying bathymetry, Coast. Eng. 18 (1992) 183-204.

[34] F. Marche, P. Bonneton, P. Fabrie, N. Seguin, Evaluation of well-balanced bore-capturing schemes for 2D wetting and drying processes, Int. J. Numer. Meth. Fluids 53 (2007) 867-894.

[35] I. K. Nikolos, A. I. Delis, An unstructured node-centered finite volume scheme for shallow water flows with wet/dry fronts over complex topography, Comput. Methods Appl. Mech. Engrg 198 (2009) 3723-3750.

[36] O. Nwogu, An alternative form of the Boussinesq equations for nearshore wave propagation, Journal of Waterway, Port, Coastal, and Ocean Engineering 119 (1994) 618-638.

[37] D. H. Peregrine, Long waves on a beach, J. Fluid Mech. 27 (1967) 815-882.

[38] P. L. Roe, Approximate Riemann solvers, parameter vectors, and difference schemes, J. Comp. Phys. 43 (1981) $357-372$.

[39] V. Roeber, K. F. Cheung, M. H. Kobayashi, Shock-capturing Boussinesq-type model for nearshore wave processes, Coast. Eng. 57 (2010) 407-423.

[40] J. B. Shiach, C. G. Mingham, A temporally second-order acurate Godunov-type scheme for solving the extended Boussinesq equations., Coast. Eng. 56 (2009) 32-45.

[41] S. Soares-Frazão, V. Guinot, A second order semi-implicit hybrid scheme for one-dimensional Boussinesq-type waves in rectangular channels, Int. J. Numer. Methods Fluids 58 (2008) 237-261.

[42] C. E. Synolakis, The run up of solitary waves, J. Fluid Mech. 185 (1987) 532-545.

[43] V. V. Titov, C. E. Synolakis, Modeling of breaking and nonbreaking long-wave evolution and runup using VTCS-2, J. Waterw., Port, Coast., Ocean Engrg. 121 (1995) 308

[44] V. V. Titov, C. E. Synolakis, Numerical modeling of tidal wave runup, J. Waterw., Port, Coast., Ocean Engrg. 124 (1998) 157-171.

[45] M. Tonelli, M. Petti, Hybrid finite-volume finite-difference scheme for 2DH improved Boussinesq equations, Coast. Eng. 56 (2009) 609-620.

[46] M. Tonelli, M. Petti, Finite volume scheme for the solution of 2D extended Boussinesq equations in the surf zone, Ocean. Eng. 37 (2010) 567-582.

[47] E. F. Toro, Shock-Capturing Methods for Free Surface Shallow Flows, Wiley and Sons, Chichester, 2001.

[48] E. F. Toro, P. Garcia-Navarro, Godunov-type methods for free-surface shallow flows: A review, J. of Hydraulic Res. 45 (2007) 736-751.

[49] G. Wei, J. T. Kirby, A time-dependent numerical code for extended Boussinesq equations, Journal of Waterway, Port, Coastal, and Ocsean Engineering 120 (1995) 251-261.

[50] S. Yamamoto, S. Kano, H. Daiguji, An efficient CFD approach for simulating unsteady hypersonic shock-shock interface flows, Computers and Fluids 27 (1998) 571-580.

[51] J. A. Zelt, The run-up of nonbreaking and breaking solitary waves, Coastal Eng. 15 (1991) 205-246.

[52] J. G. Zhou, D. M. Causon, C. J. Mingham, D. M. Ingram, The surface gradient method for the treatment of source terms in the shallow water equations, J. Comput. Phys. 168 (2001) 1-25. 